\title{
Tests to detect and inform the diagnosis of root caries (Review)
}

DOI:

10.1002/14651858.CD013806

\section{Document Version}

Final published version

Link to publication record in Manchester Research Explorer

\section{Citation for published version (APA):}

Fee, P. A., Macey, R., Walsh, T., Clarkson, J., \& Ricketts, D. (2020). Tests to detect and inform the diagnosis of root caries (Review). Cochrane Database of Systematic Reviews, (12).

https://doi.org/10.1002/14651858.CD013806

\section{Published in:}

Cochrane Database of Systematic Reviews

\section{Citing this paper}

Please note that where the full-text provided on Manchester Research Explorer is the Author Accepted Manuscript or Proof version this may differ from the final Published version. If citing, it is advised that you check and use the publisher's definitive version.

\section{General rights}

Copyright and moral rights for the publications made accessible in the Research Explorer are retained by the authors and/or other copyright owners and it is a condition of accessing publications that users recognise and abide by the legal requirements associated with these rights.

\section{Takedown policy}

If you believe that this document breaches copyright please refer to the University of Manchester's Takedown Procedures [http://man.ac.uk/04Y6Bo] or contact uml.scholarlycommunications@manchester.ac.uk providing relevant details, so we can investigate your claim.

\section{OPEN ACCESS}


Cochrane Database of Systematic Reviews

\section{Tests to detect and inform the diagnosis of root caries (Review)}

Fee PA, Macey R, Walsh T, Clarkson JE, Ricketts D

Fee PA, Macey R, Walsh T, Clarkson JE, Ricketts D.

Tests to detect and inform the diagnosis of root caries.

Cochrane Database of Systematic Reviews 2020, Issue 12. Art. No.: CD013806.

DOI: 10.1002/14651858.CD013806.

www.cochranelibrary.com 
HEADER 1

ABSTRACT

PLAIN LANGUAGE SUMMARY

SUMMARY OF FINDINGS

BACKGROUND

Figure 1.

OBJECTIVES

METHODS

RESULTS

Figure 2.

Figure 3.

Figure 4.

Figure 5.

DISCUSSION

AUTHORS' CONCLUSIONS

ACKNOWLEDGEMENTS

REFERENCES

CHARACTERISTICS OF STUDIES

ADDITIONAL TABLES

HISTORY

CONTRIBUTIONS OF AUTHORS

DECLARATIONS OF INTEREST

SOURCES OF SUPPORT

\section{TABLE OF CONTENTS}


[Diagnostic Test Accuracy Review]

\section{Tests to detect and inform the diagnosis of root caries}

Patrick A Fee ${ }^{1}$, Richard Macey ${ }^{2}$, Tanya Walsh ${ }^{3}$, Janet E Clarkson ${ }^{4}$, David Ricketts ${ }^{5}$

1Dundee Dental School, University of Dundee, Dundee, UK. 2Nuffield Department of Orthopaedics, Rheumatology and Musculoskeletal Sciences (NDORMS), University of Oxford, Oxford, UK. ${ }^{3}$ Division of Dentistry, School of Medical Sciences, Faculty of Biology, Medicine and Health, The University of Manchester, Manchester, UK. ${ }^{4}$ Division of Oral Health Sciences, Dundee Dental School, University of Dundee, Dundee, UK. ${ }^{5}$ Dundee Dental School, University of Dundee, Dundee, UK

Contact address: Tanya Walsh, tanya.walsh@manchester.ac.uk.

Editorial group: Cochrane Oral Health Group.

Publication status and date: New, published in Issue 12, 2020.

Citation: Fee PA, Macey R, Walsh T, Clarkson JE, Ricketts D. Tests to detect and inform the diagnosis of root caries. Cochrane Database of Systematic Reviews 2020, Issue 12. Art. No.: CD013806. DOI: 10.1002/14651858.CD013806.

Copyright (c) 2020 The Cochrane Collaboration. Published by John Wiley \& Sons, Ltd.

\section{A B S T R A C T}

\section{Background}

Root caries is a well-recognised disease, with increasing prevalence as populations age and retain more of their natural teeth into later life. Like coronal caries, root caries can be associated with pain, discomfort, tooth loss, and contribute significantly to poorer oral healthrelated quality of life in the elderly. Supplementing the visual-tactile examination could prove beneficial in improving the accuracy of early detection and diagnosis. The detection of root caries lesions at an early stage in the disease continuum can inform diagnosis and lead to targeted preventive therapies and lesion arrest.

\section{Objectives}

To assess the diagnostic test accuracy of index tests for the detection and diagnosis of root caries in adults, used alone or in combination with other tests.

\section{Search methods}

Cochrane Oral Health's Information Specialist undertook a search of the following databases: MEDLINE Ovid (1946 to 31 December 2018); Embase Ovid (1980 to 31 December 2018); US National Institutes of Health Ongoing Trials Register (ClinicalTrials.gov, to 31 December 2018); and the World Health Organization International Clinical Trials Registry Platform (to 31 December 2018). We studied reference lists as well as published systematic review articles.

\section{Selection criteria}

We included diagnostic accuracy study designs that compared one or more index tests (laser fluorescence, radiographs, visual examination, electronic caries monitor (ECM), transillumination), either independently or in combination, with a reference standard. This included prospective studies that evaluated the diagnostic accuracy of single index tests and studies that directly compared two or more index tests. In vitro and in vivo studies were eligible for inclusion but studies that artificially created carious lesions were excluded.

\section{Data collection and analysis}

Two review authors extracted data independently and in duplicate using a standardised data extraction and quality assessment form based on the Quality Assessment of Diagnostic Accuracy Studies 2 (QUADAS-2) specific to the review context. Estimates of diagnostic test accuracy were expressed as sensitivity and specificity with $95 \%$ confidence intervals $(\mathrm{Cl})$ for each dataset. We planned to use hierarchical models for data synthesis and explore potential sources of heterogeneity through meta-regression. 


\section{Main results}

Four cross-sectional diagnostic test accuracy studies providing eight datasets with data from 4997 root surfaces were analysed. Two in vitro studies evaluated secondary root caries lesions on extracted teeth and two in vivo studies evaluated primary root caries lesions within the oral cavity. Four studies evaluated laser fluorescence and reported estimates of sensitivity ranging from 0.50 to 0.81 and specificity ranging from 0.40 to 0.80 . Two studies evaluated radiographs and reported estimates of sensitivity ranging from 0.40 to 0.63 and specificity ranging from 0.31 to 0.80 . One study evaluated visual examination and reported sensitivity of $0.75(95 \% \mathrm{Cl} 0.48$ to 0.93 ) and specificity of 0.38 ( $95 \%$ $\mathrm{Cl} 0.14$ to 0.68 ). One study evaluated the accuracy of radiograph and visual examination in combination and reported sensitivity of 0.81 $(95 \% \mathrm{Cl} 0.54$ to 0.96$)$ and specificity of $0.54(95 \% \mathrm{Cl} 0.25$ to 0.81$)$. Given the small number of studies and important differences in the clinical and methodological characteristics of the studies we were unable to pool the results. Consequently, we were unable to formally evaluate the comparative accuracy of the different tests considered in this review. Using QUADAS- 2 we judged all four studies to be at overall high risk of bias, but only two to have applicability concerns (patient selection domain). Reasons included bias in the selection process, use of post hoc (data driven) positivity thresholds, use of an imperfect reference standard, and use of extracted teeth.

We downgraded the certainty of the evidence due to study limitations and serious imprecision of the results (downgraded two levels), and judged the certainty of the evidence to be very low.

\section{Authors' conclusions}

Visual-tactile examination is the mainstay of root caries detection and diagnosis; however, due to the paucity of the evidence base and the very low certainty of the evidence we were unable to determine the additional benefit of adjunctive diagnostic tests for the detection and diagnosis of root caries.

\section{PLAIN LANGUAGE SUMMARY}

\section{Tests to detect and inform the diagnosis of root caries}

\section{Why is it important to improve root caries detection?}

Root caries (tooth decay on the root of a tooth) is a well-recognised disease, that is on the increase as populations grow older and keep more of their natural teeth into later life. Like coronal caries (tooth decay on the crown of the tooth), root caries can be associated with pain, discomfort, and tooth loss, which can contribute to poorer oral health-related quality of life in the elderly. Detecting caries earlier can mean invasive treatment is needed, where more tooth tissue can be preserved. It could also mean less cost to the patient and to healthcare services.

\section{What is the aim of this review?}

The aim of this Cochrane Review was to find out whether any diagnostic tools could be used to support the general dentist to correctly identify root caries in adults.

Researchers in Cochrane included four studies to answer this question.

\section{What was studied in the review?}

Four studies including 4997 root surfaces were included in the review. The studies took place in Switzerland and Hong Kong, and were published between 2009 and 2016. The accuracy of laser tests was examined in four studies, two studies examined radiographs (x-rays), one study examined comprehensive visual examination, and one study examined a combined test of radiographs and visual examination.

\section{What are the main results of the review?}

All studies reported case finding (detection) rather than diagnosis that included the consideration of patient risk and history. Two studies evaluated the use of devices within the mouth, and two studies evaluated the use of devices on extracted teeth (in vitro studies). Due to the small number of studies and important differences in the setting of included studies we were unable to combine the results of the studies.

\section{How reliable are the results of the studies in this review?}

We found important study limitations in all included studies, particularly with participant enrolment which was often poorly reported. Applicability of patient selection was also of concern for two in vitro studies. For these reasons, we judged the certainty of the evidence to be very low.

\section{Who do the results of this review apply to?}

Studies included in the review were carried out in Hong Kong and Switzerland and aimed at the general dental practitioner conducting a clinical examination on adults attending a dental setting.

\section{What are the implications of this review?}


Due to the small number of studies and the very low certainty of the evidence we were unable to establish any additional benefit of diagnostic tools for the detection and diagnosis of root caries.

\section{How up-to-date is this review?}

The review authors searched for and used studies published up to 31 December 2018. 


\section{S U M MARY OF FINDINGS}

\section{Summary of findings 1 . Summary of findings table}

\begin{tabular}{|c|c|c|}
\hline Question & \multicolumn{2}{|c|}{ What is the diagnostic accuracy of index tests for the detection and diagnosis of root caries? } \\
\hline Population & \multicolumn{2}{|c|}{$\begin{array}{l}\text { Adults who present asymptomatically or are suspected of having root caries (in vivo studies); extracted teeth } \\
\text { of adults (in vitro studies). Studies which included artificially created carious lesions were excluded }\end{array}$} \\
\hline Index test & \multicolumn{2}{|c|}{$\begin{array}{l}\text { Any test for root caries detection or diagnosis. Included studies evaluated the accuracy of laser fluores- } \\
\text { cence-based devices, radiographs, visual examination, and radiograph and visual examination in combina- } \\
\text { tion }\end{array}$} \\
\hline Target condition & \multicolumn{2}{|l|}{ Root caries } \\
\hline Reference standard & \multicolumn{2}{|l|}{ Histology, visual-tactile examination } \\
\hline Action & \multicolumn{2}{|c|}{$\begin{array}{l}\text { The detection and diagnosis of early root caries lesions can provide opportunities for targeted prevention } \\
\text { and lesion arrest, and preservation of the tooth structure }\end{array}$} \\
\hline Diagnostic stage & \multicolumn{2}{|c|}{ Aimed at the general dental practitioner assessing regularly attending patients for early root caries lesions } \\
\hline $\begin{array}{l}\text { Quantity of evi- } \\
\text { dence }\end{array}$ & \multicolumn{2}{|l|}{4 studies conducted in Switzerland and Hong Kong, evaluating 4997 root surfaces } \\
\hline Findings $a$ & & $\begin{array}{l}\text { Certainty of the evi- } \\
\text { dence }\end{array}$ \\
\hline Laser fluorescence & $\begin{array}{l}4 \text { studies ( } 4997 \text { root surfaces) investigated laser fluorescence and reported esti- } \\
\text { mates of sensitivity ranging from } 0.50 \text { to } 0.81 \text { and specificity ranging from } 0.40 \text { to } \\
0.80 .2 \text { studies evaluated tests on in vivo primary root caries lesions using visual-tac- } \\
\text { tile examination as a reference standard and } 2 \text { studies evaluated tests on in vitro } \\
\text { secondary root caries lesions using histology as a reference standard }\end{array}$ & $\begin{array}{l}\oplus \odot \odot \odot \\
\text { VERY LOW }\end{array}$ \\
\hline Radiographs & $\begin{array}{l}2 \text { in vitro studies (59 root surfaces) using extracted, restored teeth and a histological } \\
\text { reference standard investigated the accuracy of radiographs. Reported estimates of } \\
\text { sensitivity ranged from } 0.40 \text { to } 0.63 \text { and specificity ranged from } 0.31 \text { to } 0.80\end{array}$ & \\
\hline Visual examination & $\begin{array}{l}1 \text { in vitro study ( } 29 \text { root surfaces) using extracted, restored teeth and a histological } \\
\text { reference standard investigated the accuracy of a visual examination and reported } \\
\text { sensitivity of } 0.75 \text { ( } 95 \% \mathrm{Cl} 0.48 \text { to } 0.93 \text { ) and specificity of } 0.38(95 \% \mathrm{Cl} 0.14 \text { to } 0.68)\end{array}$ & \\
\hline $\begin{array}{l}\text { Radiograph and vi- } \\
\text { sual examination } \\
\text { combined }\end{array}$ & $\begin{array}{l}1 \text { study ( } 29 \text { root surfaces) investigated a combined radiographic and visual examina- } \\
\text { tion and reported sensitivity of } 0.81(95 \% \mathrm{Cl} 0.54 \text { to } 0.96) \text { and specificity of } 0.54 \text { ( } 95 \% \\
\mathrm{Cl} 0.25 \text { to } 0.81)\end{array}$ & \\
\hline Quality of studies & $\begin{array}{l}\text { Using QUADAS- } 2 \text { we judged all } 4 \text { studies to be at overall high risk of bias. Reasons in- } \\
\text { cluded bias in the selection process, use of post hoc (data driven) positivity thresh- } \\
\text { olds, and the use of an imperfect reference standard. We downgraded the certain- } \\
\text { ty of the evidence due to study limitations and serious imprecision of results (down- } \\
\text { graded } 2 \text { levels), and consequently judged the certainty of the evidence to be very } \\
\text { low }\end{array}$ & \\
\hline
\end{tabular}

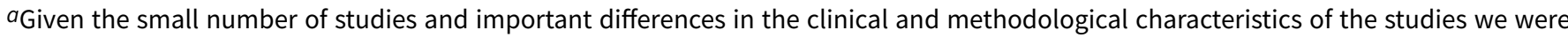
unable to pool the results of the studies. Consequently, we were unable to formally evaluate the comparative accuracy of the different tests considered in this review.

CI: confidence interval; QUADAS-2: Quality Assessment of Diagnostic Accuracy Studies 2. 


\section{B A C K G R O U N D}

Funded by the UK National Institute for Health Research (NIHR), Cochrane Oral Health $(\mathrm{COH})$ has undertaken a suite of systematic reviews on the detection and diagnosis of dental caries. The reviews follow standard Cochrane Diagnostic Test Accuracy (DTA) methodology with a generic protocol that serves as the basis for the suite of reviews (Macey 2018). This review covers tests for the detection and diagnosis of root caries.

Caries is an entire disease process, which can be stabilised and sometimes reversed if diagnosed and treated early on in the disease process (Fejerskov 2015; Pitts 2009). Whilst largely a preventable disease, the 2015 Global Burden of Disease study identified dental caries as the most prevalent, preventable condition worldwide (Kassebaum 2015; Vos 2016), affecting $60 \%$ to $90 \%$ of children and the majority of adults of the world's population (Petersen 2005). In 2010 the global prevalence of untreated caries was reported to be 2.4 billion (Kassebaum 2015; Vos 2016; World Health Organization 2017), and despite a reduction in caries in some industrialised countries, the global incidence of caries in permanent teeth has risen by $14.2 \%$ between 2005 and 2015 (Vos 2016). Root caries is defined as an area on the tooth surface, at or apical to the enamel-cementum junction, that has been affected by the caries process (Banting 2001). Root caries is however distinct from coronal caries, with significant differences regarding the morphology and structure of enamel, dentine and cementum, and differences between root and coronal caries reported in terms of the microbiology, the critical $\mathrm{pH}$ for demineralisation, and histopathology of carious lesions (Damé-Teixeira 2017).

Root caries is a well-recognised disease, with increasing prevalence as populations age and retain more of their natural teeth into later life (Adult Dental Health Survey 2009; Muller 2007; Schwendicke 2018; Slade 2014). The reported prevalence of root caries for differing populations varies widely, and ranges from $4 \%$ to $100 \%$ of the population (Christensen 2015; Fejerskov 1991). Like coronal caries, root caries can be associated with pain, discomfort, and tooth loss (Fure 1997; Slade 1997), the latter contributing most significantly to poorer oral health-related quality of life in the elderly (Slade 1996a; Slade 1996b).

Globally, dental caries is the fourth-most expensive chronic disease to treat (Petersen 2008) and there is evidence that the burden of untreated caries is now shifting from children to adults, with peaks in prevalence in early childhood, at age 25 years old and again around 70 years of age; this peak in later life occurring with the development of root caries (Kassebaum 2015).

Caries detection and diagnosis will usually be undertaken at a routine dental examination, by a general dental practitioner, in patients who are presenting asymptomatically. However, caries detection can additionally be undertaken in secondary care settings, school or community screening projects, and epidemiology or research studies (Braga 2009; Jones 2017). The traditional method of detecting root caries lesions in clinical practice is a visual-tactile examination, with lesion texture, colour, location, surface contour, cavitation, and light reflectance considered predictive of active lesions by different diagnostic criteria (Banting 2001; Ekstrand 2008; Fejerskov 1991; Lynch 1994; World Health Organization 2013). The detection of root caries at an early stage in the disease continuum can lead to targeted preventive therapies and lesion arrest, and will provide patients with the greatest chance of maintaining their teeth (Burrow 2017; Elderton 2003). Root caries lesions detected at an early, non-cavitated stage respond more favourably to preventive interventions compared to cavitated lesions and have a higher rate of remineralisation than advanced lesions (Baysan 2001; DePaola 1993; Ekstrand 2013). However, the detection of root caries lesions at an early stage can be more complex compared to coronal caries as the early white spot lesion, present in enamel, is not evident on root surfaces (Pretty 2017). Instead the only reported signs of early root caries are the softening of the root dentine surface and a slight change of colour to light brown. Restorative treatment is typically required for more extensive, cavitated lesions.

Advances in technology have led to the development of alternative methods of caries detection to support the traditional conventional oral examination. These methods include digital radiography, fluorescence- or transillumination-based technologies, and electrical conductance devices. These methods, when considered in the context of other diagnostic information offer the potential to support the diagnosis of root caries at an early stage of decay. This could afford dental patients the opportunity of less invasive treatment with less destruction of tooth tissue and potentially result in a reduced cost of care to the patient and to healthcare services.

\section{Target condition being diagnosed}

The term dental caries is used to describe the mechanisms and symptoms of the breakdown on the tooth surface which result from an imbalance in the activity within the biofilm (or dental plaque) within the oral cavity (Kidd 2016). This imbalance is especially related to $\mathrm{pH}$ levels on the surface of the tooth which are readily affected by the consumption of fermentable carbohydrate which, in the presence of cariogenic root biofilm, is converted into organic acids. Disease progression can be moderated by disruption of the biofilm with improved oral hygiene behaviours and by the influx of fluoride through toothpaste and other available fluoride sources. Ultimately, if this does not occur, carious lesions may develop and destroy the structure of the tooth.

The intraoral distribution of root caries differs between studies but the mandibular molars and premolars appear to be the most commonly affected, followed by the maxillary canines and incisors while the mandibular incisors are the least commonly affected (Katz 1982). The approximal and buccal surfaces of the mandibular posterior teeth are the most commonly affected root surfaces (Katz 1982). Root caries lesions presenting at an early stage have the potential to arrest or remineralise, whereas advanced, cavitated lesions where regular patient-performed plaque removal cannot be consistently achieved will require restoration (Burrow 2017; Maltz 2017). Whereas active root caries lesions are described as soft in texture on gentle probing, an arrested lesion is indicated by signs of a hardened dentine surface not easily penetrated upon light force from a blunt probe and a shiny surface (Banting 2001; Pretty 2017; Rodrigues 2011). Uncertainty exists regarding the use of lesion colour to determine lesion activity and while once thought highly predictive - active lesions light brown or yellowish and arrested lesions dark brown or black - is now disregarded by some authorities (Pretty 2017).

The assessment of caries severity that has traditionally been used in epidemiological and research studies has employed some variant of the decayed, missing, and filled teeth (DMFT) scale. 
Assessment of root caries severity has commonly used the root decayed and filled surfaces (RDFS) scale, the percentage of the population with any root caries lesions, or the average number of decayed root surfaces per person. Within the D (decayed) component of DMFT there are four clinically detectable thresholds applied as indicators for diagnosis and treatment planning, often labelled as $D_{1}, D_{2}, D_{3}$, and $D_{4}$ (Anaise 1984) (Additional Table 1 ). The $D_{1}$ and $D_{2}$ thresholds only pertain to coronal caries where enamel caries is concerned. Typically the $D_{3}$ threshold has been used to determine the presence of caries (Pitts 1988; Shoaib 2009). As the $D_{3}$ threshold describes caries extending into dentine, some epidemiological studies and clinical trials combine coronal caries lesions progressing into dentine with root caries lesions at this threshold when presenting results, while other studies report coronal caries lesions and root caries lesions separately.

\section{Treatment of root caries}

There are varied treatment options available for the treatment of root caries, dependent upon the extent, cavitation, and cleansability of the lesion. Early root caries lesions can be treated without surgical intervention using preventive and remineralising approaches such as plaque control, dietary advice, and application of fluoride (Burrow 2017; Maltz 2017). Non-invasive interventions in the form of 5000 parts per million (ppm) fluoride toothpaste and professionally applied sodium fluoride varnish, chlorhexidine varnish, or silver diamine fluoride have been recommended to arrest non-cavitated or cavitated root caries lesions (Slayton 2018) and seem to be more efficacious in arresting root caries lesions than conventional fluoride toothpaste or placebo interventions (MeyerLueckel 2019).

Minimally invasive treatments include removal of overlying unsupported enamel in cavitated lesions to expose the dentine lesion and enable biofilm removal and ultimately lesion remineralisation for cavitated lesions close to the enamelcementum junction. Deeper or more widespread non-cleansable lesions may require restoration. It is important to note however, the high failure rates of root surface restorations compared with coronal restorations (Hayes 2016; Meyer-Lueckel 2019), supporting the need to consider minimally invasive treatment options in the management of root caries lesions.

A caries management pathway, informed by diagnostic information, can be beneficial in guiding the clinician towards prevention or a treatment plan. One recently developed care pathway is the International Caries Classification and Management System $\left(\right.$ ICCMS $^{\mathrm{TM}}$ ) (Ismail 2015). This system provides guidance on the staging of coronal and root caries lesions and presents three forms of management in the care pathway:

1. when dentition is sound the clinician proceeds with preventative strategies to prevent sound surfaces from developing caries;

2. non-invasive treatment of the lesion to arrest the decay process and encourage remineralisation, preventing initial lesions from progressing to cavitated decay; and

3. management of more severe caries through excavation and restoration or potentially extraction.

The ICCMS pathway is perhaps more relevant to coronal caries but the principles outlined can be applied to decision making in the management of root caries. At the core of this care pathway is the ability to detect early caries accurately and optimise preventative strategies. The detection and diagnosis of early root caries remains challenging and more complex than that of enamel caries, where white spot lesions can indicate early demineralisation (Pretty 2017).

The likelihood of undiagnosed early disease is high, and opportunities for early intervention to arrest lesion progression or remineralise root caries lesions may be missed, resulting in lesion progression requiring more invasive treatment.

\section{Index test(s)}

A visual-tactile examination is the predominant means of detecting root caries lesions in clinical practice, relying on a combination of the visual appearance and texture on gentle probing of the clean, dry root surface. Examination can be completed and interpreted quickly with minimal invasion and little cost except clinician training and time. However, detection of early or approximal lesions is often difficult to observe on visual-tactile examination. Longitudinal monitoring of root caries lesions is also difficult with visual-tactile methods. A number of caries detection methods exist to support the conventional clinical examination in diagnosing disease at different stages of the care pathway. These include.

\section{- Radiography. \\ - Fluorescence. \\ - Electrical conductance. \\ - Fibre-optic transillumination.}

Bitewing radiographs are the most commonly used radiography method in detection of caries lesions. Other methods include: subtraction radiographs which provide a semi-automated method for monitoring progression of lesions (Ellwood 1997; Wenzel 2000) and cone beam computed technology (CBCT), which provides a three-dimensional image which appears to offer great potential for aiding diagnosis with increased levels of radiation (Horner 2009). Bitewing radiographs may aid in the detection of approximal cavitated root caries lesions difficult to detect with visual-tactile examination alone (Rodrigues 2011), however limitations exist in the detection of early, non-cavitated lesions, and cervical burnout presenting as proximal radiolucency can complicate accurate detection of root caries radiographically. There is a small but real risk over patient exposure to ionising radiation, which has to be balanced with the patient's age, caries risk, and time since previous radiograph (Pitts 2017).

Caries-induced changes in teeth alter the characteristics of its structure, causing diseased teeth to respond differently to sound teeth when exposed to light-inducing fluorescence. There is potential for mineral loss to be quantified and used to aid the diagnostic decision and treatment pathway (Angmar-Månsson 2001; Matos 2011; Wicht 2002). Fluorescence is typically divided into laser fluorescence and light fluorescence (i.e. DIAGNOdent type devices and quantitative light-induced fluorescence (QLF) type devices). Fluorescence tests offer the potential to aid clinicians in identifying early carious lesions which may be otherwise unobservable in a visual-tactile examination alone. There is however, uncertainty regarding the accuracy of fluorescence devices in their ability to detect sound and carious root surfaces.

Fibre-optic transillumination (FOTI) uses a light emitted from a handheld device which when placed directly onto the tooth 
illuminates the tooth (Pretty 2006). Any demineralisation should appear as shadows in the tooth due to the disruption of the tooth's structure due to the caries process. FOTI presents the potential to aid detection of early approximal caries (Davies 2001), but there is uncertainty regarding the accuracy of these devices in their ability to detect sound and carious root surfaces.

Demineralisation of tooth structure is reported to have an effect on the tooth's electrical conductance. This is measured by placing a probe on the tooth which measures any potentially higher conductivity which occurs due to carious lesions being filled with saliva (Tam 2001). Electrical conductance tests therefore provide the potential to identify changes in tooth characteristics that are otherwise unobservable in a visual-tactile examination. There is however, uncertainty regarding the accuracy of electrical conductance devices in their ability to detect sound and carious root surfaces, complicated by the necessity to place the probe tip in an identical location relative to the caries lesion to produce a reproducible result.

A summary of characteristics of index tests and their advantages and disadvantages to consider is included in Additional Table 2.

\section{Clinical pathway}

The process proceeding from a dental patient attending for a routine examination and a caries assessment being undertaken potentially has four intertwined stages: screening, detection, diagnosis, and treatment planning. If the presenting patient is seemingly asymptomatic then this could be viewed as a screening exercise, as the clinician is seeking to establish who probably has caries and who is healthy (Wilson 1968). Detection is a more reasonable description of an initial examination where the clinician aims to establish the true presence or absence of disease. Since caries is a dynamic process the detection of the disease at a single time point is not sufficient to inform the future care of the patient, with longitudinal monitoring of lesion progression over time necessary to confirm lesion activity and therefore reach a diagnosis. This diagnosis then feeds into a caries management pathway once the patient's history, personal oral care, and risk factors have been considered. The International Caries Classification and Management System (ICCMS ${ }^{\mathrm{TM}}$ ) has been developed to address the need for guidance to support diagnosis and the subsequent decision-making process to use preventative measures and minimise invasive treatment (Ismail 2015).

Figure 1 presents the key elements of the ICCMS process and this review could inform the process at 'Keystone 3' where diagnosis is an indefinable component. The ICCMS has been further developed, Caries Care International, for use in primary care (Martignon 2019).

Figure 1. Keystones of the International Caries Classification and Management System (ICCMS ${ }^{\mathrm{TM}}$ ). Copyright $\odot 2018$ Ismail AI, Pitts NB, Tellez M. The International Caries Classification and Management System (ICCMS ${ }^{\mathrm{TM}}$ ) an example of a caries management pathway. BMC Oral Health 2015;15(Suppl 1):S9. Reproduced with permission.

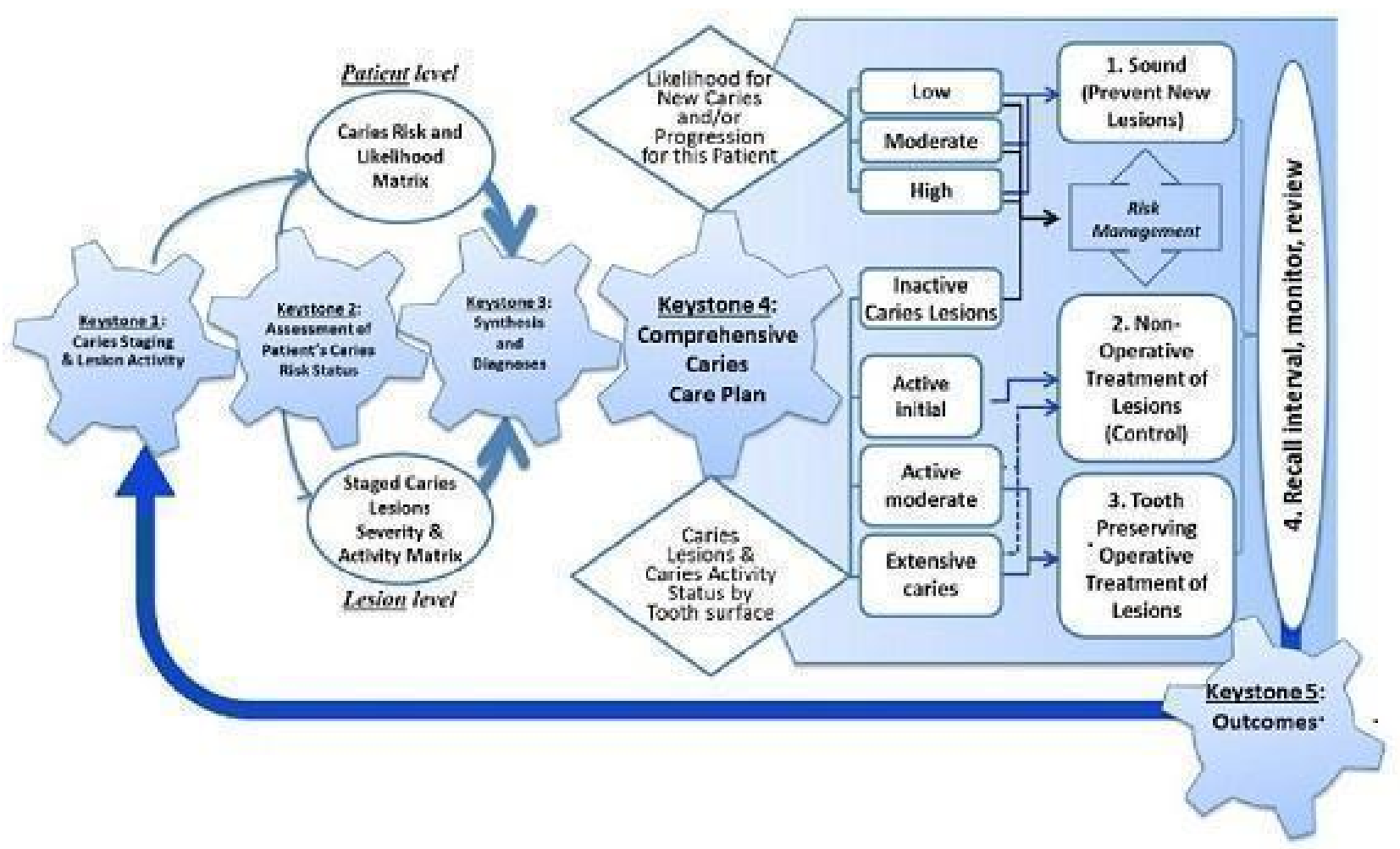




\section{Role of index test}

As the visual-tactile examination is the mainstay of a clinical examination it is unlikely that any of the index tests under evaluation in this review would be used as a complete replacement for the detection and diagnosis of root caries. Supplementing the visual-tactile examination could prove beneficial in improving the accuracy of detection as opposed to the visual-tactile examination alone. The index tests could also have a triage role in assisting dental personnel to more accurately assess signs of uncertain clinical significance. The information from caries detection (including assessment of severity of disease) is an integral component of diagnosis, which additionally incorporates patient history, risk factors, and treatment planning protocols.

\section{Rationale}

To our knowledge, this is the first diagnostic test accuracy review to report root caries lesions separately from coronal caries lesions. A systematic review by Brouwer investigated the accuracy of diagnostic tests in the detection of secondary caries lesions (Brouwer 2016). This review included two studies providing data for root caries lesions which was combined with data provided for coronal caries. Root caries is significantly different from coronal caries histopathologically. Given the diagnostic specificities of root caries (Damé-Teixeira 2017), particularly the presence of enamel on coronal surfaces allowing early enamel caries detection as a white spot lesion (Pretty 2017), and the absence of both on root surfaces complicating early detection, analysis of the accuracy of tests for the detection of root caries independent of coronal caries is more appropriate and relevant to clinical practice.

We aimed to build upon existing research in this area by: assessing the accuracy of tests for the detection of root caries lesions independent of coronal caries, expanding the search strategy to capture all relevant evidence, and assessing the body of evidence using GRADE (Schünemann 2020; Schünemann 2020a) to facilitate the production of evidence summaries and evidence to decision criteria.

\section{OB JECTIVES}

To assess the diagnostic test accuracy of index tests for the detection and diagnosis of root caries in adults, used alone or in combination with other tests.

We aimed to evaluate the comparative accuracy of all index tests incorporating: visual or visual-tactile examination, radiography, fluorescence, electrical conductance, and fibre-optic transillumination.

The specific research questions addressed in this Cochrane Review were.

- What is the diagnostic test accuracy of each of the index tests compared to an appropriate reference standard for detecting and diagnosing early decay on root surfaces?

- Do measures of sensitivity and specificity for single tests differ from the sensitivity and specificity of tests used in combination? Is there a benefit to using more than one index test as opposed to a single test?

- What is the comparative diagnostic test accuracy of the different index tests?

\section{Secondary objectives}

We aimed to investigate the following potential sources of heterogeneity:

- in vitro or in vivo studies;

- tooth surface;

- participants or teeth with root surface restorations (secondary caries);

- prevalence of root caries;

- choice of reference standard used (histology or enhanced visualtactile examination, or a composite reference standard).

\section{METHODS}

\section{Criteria for considering studies for this review}

\section{Types of studies}

We considered diagnostic accuracy study designs that were:

- studies with a single set of inclusion criteria that compared a diagnostic test with a reference standard. We included prospective studies that evaluated the diagnostic accuracy of single index tests, and studies that directly compared two or more index tests;

- studies that evaluated tests alone, in comparison to another test, multiple tests, or test combinations;

- randomised controlled trials (RCTs) of the diagnostic test accuracy of one or more index tests in comparison, or versus a no test option;

- 'case-control' type accuracy studies where different sets of criteria were used to recruit those with or without the target condition (although prone to bias, some innovative tests may be identifiable through this design only);

- studies reporting at both the patient and tooth or tooth surface level were included, however only studies that reported at the tooth surface level would be included in the primary analysis.

In vitro (based on extracted teeth) and in vivo (clinical setting, teeth in situ when assessed by the index test) were eligible for inclusion. In vitro studies typically involve a reference standard of histology but lack clinical generalisability; in vivo studies benefit from assessment of teeth in situ and are more representative of the true clinical context of assessment within the oral cavity.

Studies where artificially created carious lesions were used in the testing procedure, an index test was used during the excavation of root caries to ascertain the optimum depth of excavation, or where data were not reported in a format that allowed construction of $a 2 x$ 2 contingency table were excluded. We also excluded studies which only reported the diagnostic test accuracy results for coronal and root caries combined.

\section{Participants}

The selection of patients has a fundamental effect on an index test's ability to detect caries. It was therefore essential that the disease stages of sound root surfaces and early root caries lesions be represented in the sample. Studies including adult participants seemingly asymptomatic for root caries were therefore included, while studies that explicitly recruited participants with root caries lesions were excluded as were those with participants referred to 
secondary care for restorative treatment, as there is a likelihood that advanced cavitated root caries lesions will be present and readily detectable without the need for the index tests investigated in this review, potentially overestimating the ability of an index test to accurately detect disease.

\section{Index tests}

- Visual or visual-tactile examination according to detailed criteria and indices (e.g. World Health Organization (WHO)) (where tactile infers the use of a probe to assess lesion texture).

- Intra and extraoral radiographs, using conventional film and digital imaging.

- Fluorescence-based devices including laser-based detection and quantitative light-induced fluorescence (QLF).

- Electrical conductance.

- Fibre-optic transillumination (including white light scattering and near infrared).

- Any new, innovative test that does not fit within the other criteria.

These index tests were conducted on intact teeth and could be used as an adjunct to or replacement for aspects of the current examination. The intention was to assess the index tests in isolation where possible otherwise the result of one index test may influence another, however where multiple index tests were used as a combined index test and the individual tests could not be isolated these were reported as a separate 'combined test' group.

Where studies used multiple tips on the same fluorescence device, the most appropriate tip to the research question was selected. Where studies performed index tests on the same participants prior to and following professional cleaning or calculus removal, the results following calculus removal have been selected.

\section{Target conditions}

Root caries: non-cavitated or cavitated caries lesions affecting the root surface on smooth and approximal root surfaces.

\section{Reference standards}

The sole method of achieving a true diagnosis of caries presence and levels is to extract and section a tooth and perform a histological assessment (Carvalho 2017; Kidd 2004). This is not feasible for disease-free teeth. The only scenario where histology is viable for studies undertaken in a primary or secondary dental care setting would be where a tooth has been identified as requiring extraction (ideally for a non-caries reason, such as orthodontic extraction or as a result of periodontal disease) and the index test could be applied within the oral cavity prior to extraction of the tooth, followed by the histology as a reference standard. However, teeth extracted for orthodontic reasons are unlikely to have root caries as by far the majority of orthodontic treatment occurs in children or adolescents or younger adults with little exposure of the root surface.

Alternatives to extraction and histological assessment are operative exploration, where caries is removed with a dental bur (drill) in preparation for a restoration and the depth of decay is recorded and reported. This would be acceptable as a reference standard for patients with caries requiring restoration, but would not be ethical for caries-free patients or those with early root caries lesions since non-restorative treatment could be provided. A different reference standard would be required for these early lesions, namely visual-tactile examination, while dental radiographs are of little value in the detection of early root caries lesions (Pretty 2017).

Therefore, in this analysis, acceptable reference standards were histology or enhanced visual-tactile examination, with visualtactile examination also included as an index test only where histological examination was used as the reference standard.

\section{Search methods for identification of studies}

\section{Electronic searches}

Cochrane Oral Health's Information Specialist conducted systematic searches in the following databases without language or publication status restrictions:

- MEDLINE Ovid (1946 to 31 December 2018) (Appendix 1);

- Embase Ovid (1980 to 31 December 2018) (Appendix 2).

\section{Searching other resources}

The following trial registries were searched for ongoing studies:

- US National Institutes of Health Ongoing Trials Register ClinicalTrials.gov (clinicaltrials.gov; searched 31 December 2018) (Appendix 3);

- World Health Organization International Clinical Trials Registry Platform (apps.who.int/trialsearch; searched 31 December 2018) (Appendix 4).

We searched for additional studies not identified in the electronic searches by manually searching the reference lists of included studies, previously published systematic reviews, and relevant articles.

We checked that none of the included studies had been retracted due to error or fraud.

\section{Data collection and analysis}

\section{Selection of studies}

Two review authors (Patrick Fee (PF), Richard Macey (RM)) independently screened and assessed the results of all searches for inclusion. Any disagreements were resolved through discussion and, where necessary, consultation with another member of the author team. Where possible, studies that met the eligibility criteria but did not report the data in the format of a $2 \times 2$ contingency table were contacted and the required data requested. If no data were obtained then the study was excluded. An adapted PRISMA flowchart (McInnes 2018) was used to report the study selection process.

\section{Data extraction and management}

Two review authors extracted data independently and in duplicate using a piloted study data extraction form. Disagreements were resolved through discussion with other members of the review team.

We recorded the following data for each study: 
- sample characteristics (age, sex, ethnicity, number of patients/ carious lesions, lesion location, disease prevalence, presence of restorations);

- setting (country, type of facility);

- the type of index test(s) used (category (i.e. fluorescence, radiographs, visual), name, conditions (i.e. clean/dried teeth), positivity threshold);

- study information (design, reference standard, case definition, training and calibration of personnel);

- study results (true positive, true negative, false positive, false negative, any equivocal results).

\section{Assessment of methodological quality}

The Quality Assessment of Diagnostic Accuracy Studies 2 (QUADAS-2) was used to assess the applicability and risk of bias of the eligible primary diagnostic studies over the four domains of participant selection, index test, reference standard, and flow and timing (Whiting 2011), tailored for this review. Review specific descriptions of how the QUADAS-2 items were contextualised and implemented are detailed in the accompanying checklist (Additional Table 3).

A 'Risk of bias' judgement ('high', 'low', or 'unclear') was made for each domain. Where the answers to all signalling questions within a domain were judged as 'yes' (indicating low risk of bias for each question) then the domain was judged to be at low risk of bias. If any signalling question was judged as 'no', indicating a high risk of bias, the domain was judged to be at high risk of bias. Similarly, if any signalling question was judged as 'unclear', indicating an unclear risk of bias, the domain was judged to be at unclear risk of bias. Concerns regarding applicability were then completed for participant selection, index test, and reference standard domains and elements in the consideration of each are detailed below.

\section{Participant selection domain}

It was acceptable for studies to focus on one particular surface (smooth/approximal) or specific age group (e.g. elderly). All studies should have clearly reported the methods used to select teeth, ideally a random or consecutive selection would be used and the procedure clearly reported. Inappropriate exclusion may lead to an over or under estimation of the test's ability to detect disease, thus affecting the internal validity of the study. In addition the severity of disease (cavitated or non-cavitated) in the study sample and relevant prevalence should be clearly reported. This information was used to inform judgements regarding the applicability of a test to a wider population.

Study results should be reported at a tooth or surface level, as opposed to patient level, to avoid the potential for the index test and reference standard to report on different sites within the mouth.

\section{Index test domain}

The nature of the index tests and the visual presentation of the disease mean that it should be feasible to ensure that the index test is conducted prior to the reference standard. The visual, fluorescence, fibre optic, and radiography tests should be completed before the extraction of a tooth for any histological analysis, or before in situ excavation of a tooth is undertaken. This sequence of testing will ensure that the results of the index test are not influenced by the results of the reference standard. To minimise potential for bias, separate examiners should be utilised for index test and reference standard. Participants recruited to the study should be reflective of disease in the population. For example, in studies investigating asymptomatic patients at a screening level, then early stages of disease may be of importance and a threshold of non-cavitated root caries will be of greater relevance than cavitated root caries lesions. It is unlikely that studies will have utilised multiple index test examiners or where they have it is probable that they each score all of the thresholds and are included for validation of the test. However, the inclusion of a question here will allow the identification of studies that have achieved this and inform the future discussions.

The threshold of disease positive and negative should be presented prior to analysis, ideally by using the manufacturers recommended settings or thresholds recommended by previously validated studies. Some studies may calculate and report at thresholds that optimise values of sensitivity and specificity but this introduces bias.

\section{Reference standard domain}

If the reference standard was a visual-tactile examination then it should be completed by an examiner different to the index test, as the subjectivity of this type of reference standard could be compromised by knowledge of the index test results. An exception was built in for this signalling question because where the tooth has been extracted, sectioned, and prepared for histological evaluation it is extremely unlikely that the examiner would be able to recall the specific tooth or participant and the results from the index test results. Time delays between index test and reference standard should be under three months for in vivo studies.

Ideally, each participating tooth or patient within a study should receive the same reference standard. This is possible in the in vitro setting as each selected tooth can undergo histological analysis. In vitro studies may apply the same reference standard to all participants by using visual-tactile examination. If a study allocated participants or specific teeth to different reference tests then reasons for this allocation should have been clearly reported. All reference standards should have been completed without knowledge of the index test results.

\section{Flow and timing domain}

Ideally, the index test should be conducted prior to the reference standard. In in vivo studies, the time between index test and reference standard should be less than three months. Caries is a slowly progressing disease so minimal changes should be experienced within this time frame. All observations should receive both an index test and reference standard. Some studies report teeth having an index test but not a reference standard; if a reason is clearly reported, such as teeth being damaged during histological sectioning, then this would not influence the risk of bias decision.

\section{Statistical analysis and data synthesis}

The threshold of interest was between sound root surfaces and those with either cavitated or non-cavitated root caries lesions. Estimates of diagnostic test accuracy were expressed as sensitivity and specificity with $95 \%$ confidence intervals (Cls) for each study and for each available dataset if multiple index tests were evaluated within the same study. This information was displayed 
as coupled forest plots, and plotted as summary receiver operating characteristic (SROC) plots, displaying the sensitivity-specificity points for each study. When there were two or more test results reported in the same study, we included them as separate datasets, since the unit of analysis was the test result.

We planned to use hierarchical models for data synthesis. Where a consistent positivity threshold was reported across the studies we planned to combine the results of studies for each index test using a hierarchical bivariate approach to estimate the expected values of sensitivity and specificity (Macaskill 2010; Reitsma 2005). Where a common threshold is difficult to apply, we planned to produce a summary curve using a hierarchical summary receiver operating characteristic (HSROC) model (Rutter 2001).

As per the review questions, we planned to explore the use of different index tests for different purposes (detection and diagnosis), as an adjunct to or independent of a conventional oral examination.

\section{Investigations of heterogeneity}

If sufficient numbers of studies allowed, meta-regression analyses were planned to explore the possible sources of heterogeneity. We planned to undertake formal model comparisons using a likelihood ratio $\mathrm{Chi}^{2}$ statistic to determine the statistical significance of adding one or more potential sources of heterogeneity (covariates) to the hierarchical model.

Potential sources of heterogeneity specified a priori were:

- in vitro or in vivo studies;

- tooth surface;

- participants or teeth with root surface restorations (secondary caries);

- prevalence of root caries;

- choice of reference standard used (histology or visual-tactile examination).

\section{Sensitivity analyses}

We planned to undertake sensitivity analyses restricting the analysis to studies with:

- low risk of bias on their inclusion criteria for caries threshold;

- low prevalence of dentine caries (i.e. less than 35\%);

- low risk of bias for an index test;

- low risk of bias for a reference standard.

\section{Assessment of reporting bias}

We did not examine reporting or publication bias in this review as current quantitative methods are not well established for diagnostic test accuracy studies and may lead to uncertainty and misleading results from funnel plots (Deeks 2005; Leeflang 2008).

\section{Presentation of main results}

We reported our results following GRADE methods (Schünemann 2020; Schünemann 2020a), and using the GRADEPro online tool (www.guidelinedevelopment.org). To enhance readability and understanding, we presented test accuracy results in natural frequencies to indicate numbers of false positives and false negatives where appropriate. The certainty of the body of evidence was assessed with reference to the overall risk of bias of the included studies, the directness of the evidence, the inconsistency of the results, the precision of the estimates, and the risk of publication bias; these have been considered narratively where statistical methods were not available. We categorised the certainty of the body of evidence as high, moderate, low, or very low.

\section{RE S U L T S}

\section{Results of the search}

The search identified a total of 17,985 records through database searching and a further two records were identified through reference list citation searching. The large number of records identified being a result of conducting an overarching search for the suite of Cochrane Oral Health Reviews of diagnostic test accuracy on the detection and diagnosis of dental caries and then restricted to the target condition of root caries. Of the 13,452 records remaining after the removal of duplicates, we discarded 13,433 from their titles and abstracts, in accordance with the eligibility criteria. The remaining 19 studies were assessed from the full published paper.

Fifteen studies were excluded from this review, reasons are provided in the Characteristics of excluded studies table. The most common reasons for exclusion were studies where index tests were investigated for correlation with lesion characteristics but with no reference standard, i.e. they were comparative rather than diagnostic test accuracy studies. Other common reasons for exclusion were studies that only included participants or teeth with root caries where no sound teeth were included, or where data were not presented in a form to allow a $2 \times 2$ table to be constructed.

Four cross-sectional diagnostic test accuracy studies, published between 2009 and 2016, were eligible for inclusion (Figure 2). Two studies were completed in Hong Kong (Zhang 2009; Zhang 2016) in a community setting of elders over 60 years old, and two in Switzerland using extracted teeth with a Class II amalgam restoration (Neuhaus 2012), and a Class II resin composite restoration (Rodrigues 2010). The four index tests assessed in these studies were visual examination (Rodrigues 2010), radiographic examination (Neuhaus 2012; Rodrigues 2010), visual and radiographic examination in combination (Rodrigues 2010), and laser fluorescence (Neuhaus 2012; Rodrigues 2010; Zhang 2009; Zhang 2016). The included studies provided eight datasets evaluating: one index test against a reference standard (Zhang 2009; Zhang 2016), two index tests (Zhang 2009; Zhang 2016), and four index tests (Rodrigues 2010). The four included studies therefore provided eight datasets which evaluated 4997 root surfaces. Two studies investigated diagnostic performance of tests on approximal surfaces (Neuhaus 2012; Rodrigues 2010), one study investigated buccal and lingual surfaces (Zhang 2009), and one study did not report the surfaces under investigation (Zhang 2016). In all four studies root surfaces underwent removal of calculus with a dental scaler prior to index tests or reference standard. The prevalence of root caries ranged from $5 \%$ to $67 \%$. Two studies reported using histological assessment as the reference standard (Neuhaus 2012; Rodrigues 2010) and two studies reported using visual-tactile assessment as the reference standard (Zhang 2009; Zhang 2016). 
Figure 2. Study flow diagram.

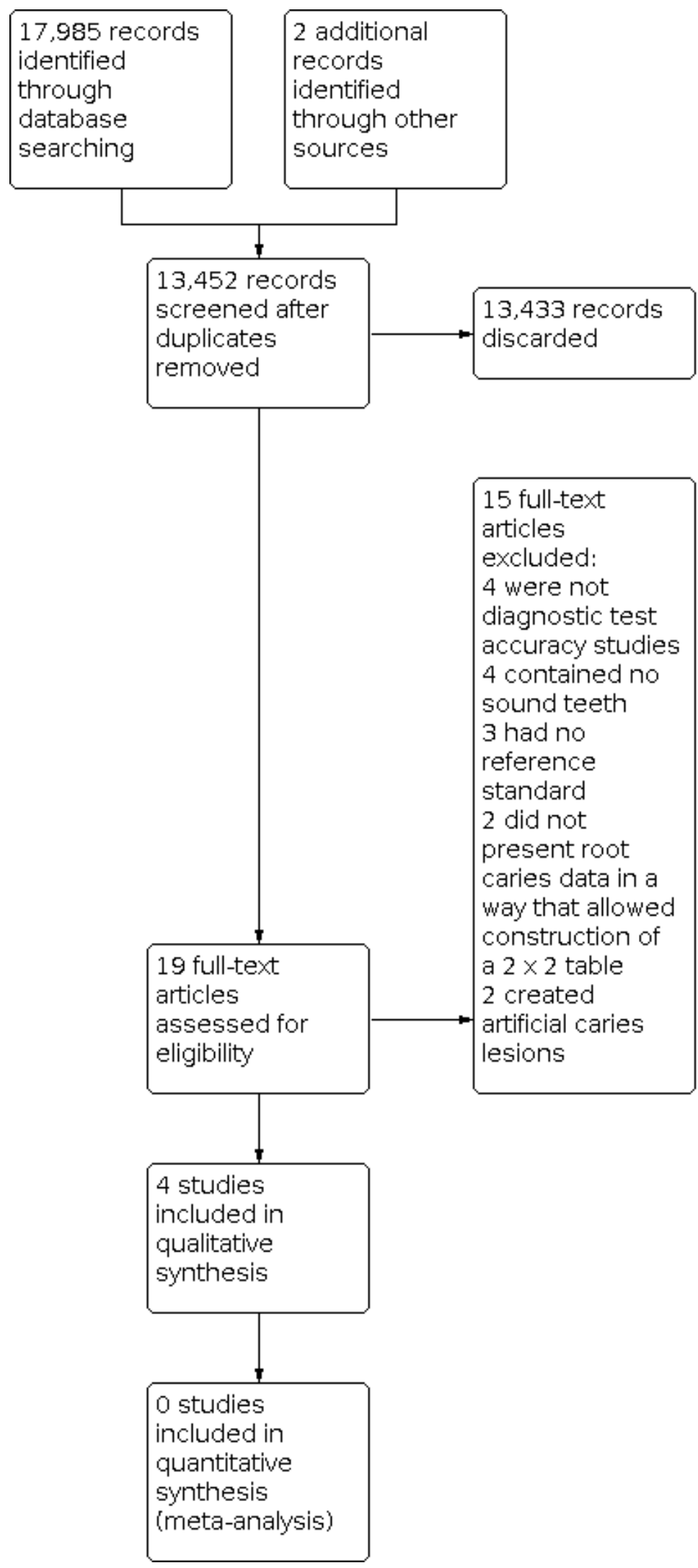




\section{Laser fluorescence}

- DIAGNOdent: two datasets evaluated a total of 4938 tooth surfaces (Zhang 2009; Zhang 2016). Both studies were carried out with adults over 60 years old residing in a community setting. Both studies used a threshold which was calculated within the study to determine the presence of root caries. The prevalence of root caries in studies investigating DIAGNOdent were $5 \%$ and $18 \%$. Both studies used visual-tactile assessment as the reference standard and both studies used the same clinical criteria to define root caries based on lesion texture (active caries: soft upon light probing, inactive caries: smooth, and hard upon light probing) (World Health Organization 1997; World Health Organization 2013) and colour (active caries: yellowish or light brownish, inactive caries: darkly discoloured) (Fejerskov 1991). One study assessed buccal and lingual surfaces (Zhang 2009), while one study did not report the root surfaces assessed (Zhang 2016). One study included only unrestored teeth (Zhang 2016) and one study did not report the restorative status of included teeth (Zhang 2009). In both studies all participants received a dental scaling prior to index or reference standard test.

- DIAGNOdent pen: two datasets evaluated a total of 59 tooth surfaces (Neuhaus 2012; Rodrigues 2010). Both were in vitro studies evaluating the approximal surfaces of extracted teeth restored with amalgam restorations or one with resin composite restorations. One study used a pre-specified threshold of a fluorescence value of 22 to determine the presence of root caries (Neuhaus 2012). One study used an optimum threshold calculated within the study to determine the presence of root caries (Rodrigues 2010). One study used a wedge-shaped sapphire fibre tip of $1.1 \mathrm{~mm}$ width, and one study used a tapered wedge-shaped tip of $0.7 \mathrm{~mm}$ width. Both studies were performed using extracted teeth mounted in pairs between two sound teeth, whose roots were embedded in composite resin, in order to obtain a tight contact. Marginal calculus was removed using a scaler prior to index and reference tests. The prevalence of root caries in studies investigating DIAGNOdent pen were $55 \%$ and $67 \%$. Both studies used histology as the reference standard. Hardness measurements of the histological specimens were performed to aid the histological classification in cases of doubt. One study used a hardness threshold of below 70 Knoop diamond (KNH) to define root caries (Rodrigues 2010) and one study used a threshold of greater than $10 \mathrm{KNH}$ levels below the baseline $\mathrm{KNH}$ value to define root caries (Neuhaus 2012).

\section{Radiograph examination}

Two datasets from two studies evaluated the diagnostic accuracy of bitewing radiographs on a total of 59 approximal tooth surfaces (Neuhaus 2012; Rodrigues 2010).

In both studies the diagnostic accuracy of bitewing radiographs for coronal and root caries was investigated and thresholds for radiographic diagnosis used the same scoring system: no radiolucency, radiolucency in enamel, and radiolucency in dentine. Both studies were performed using extracted teeth mounted in pairs between two sound teeth, whose roots were embedded in composite resin, in order to obtain a tight contact, and a 5$\mathrm{mm}$-wide plastic mould close to the object on the focus side to simulate soft tissues. Marginal calculus was removed using a scaler prior to index and reference tests. The prevalence in the two studies was $55 \%$ and $67 \%$. Both studies used histology as the reference standard, both assessed restored approximal surfaces one with amalgam restorations (Neuhaus 2012) and one with resin composite restorations (Rodrigues 2010).

\section{Visual examination}

One dataset from one study (Rodrigues 2010) evaluated the diagnostic accuracy of visual examination in 29 approximal root surfaces of extracted molar teeth restored with resin composite. The approximal surfaces observed were classified according to the presence of visible marginal colour changes surrounding the restoration site, ditches, or cavities. Each surface was scored as sound or carious. Extracted teeth were mounted in pairs between two sound teeth, whose roots were embedded in composite resin, in order to obtain a tight contact. Marginal calculus was removed using a scaler prior to index and reference tests. The prevalence of root caries in this study was 55\%. The reference standard was histology.

\section{Radiograph and visual examination combined}

One dataset from one study (Rodrigues 2010) evaluated the diagnostic accuracy of visual examination in 29 approximal root surfaces of extracted molar teeth restored with resin composite (study characteristics described above).

\section{Methodological quality of included studies}

The individual assessment for each of the included studies is illustrated in Figure 3, and summarised in Figure 4. No studies were considered to be at low risk of bias across all the domains. Patient selection was considered to be at low risk of bias in only one study (Zhang 2009) where that study stated that participants were recruited consecutively. Three studies selected the participants or teeth from an available population, without consecutive or random recruitment, and were judged to be at high risk of bias. 
Figure 3. Risk of bias and applicability concerns summary: review authors' judgements about each domain for each included study.

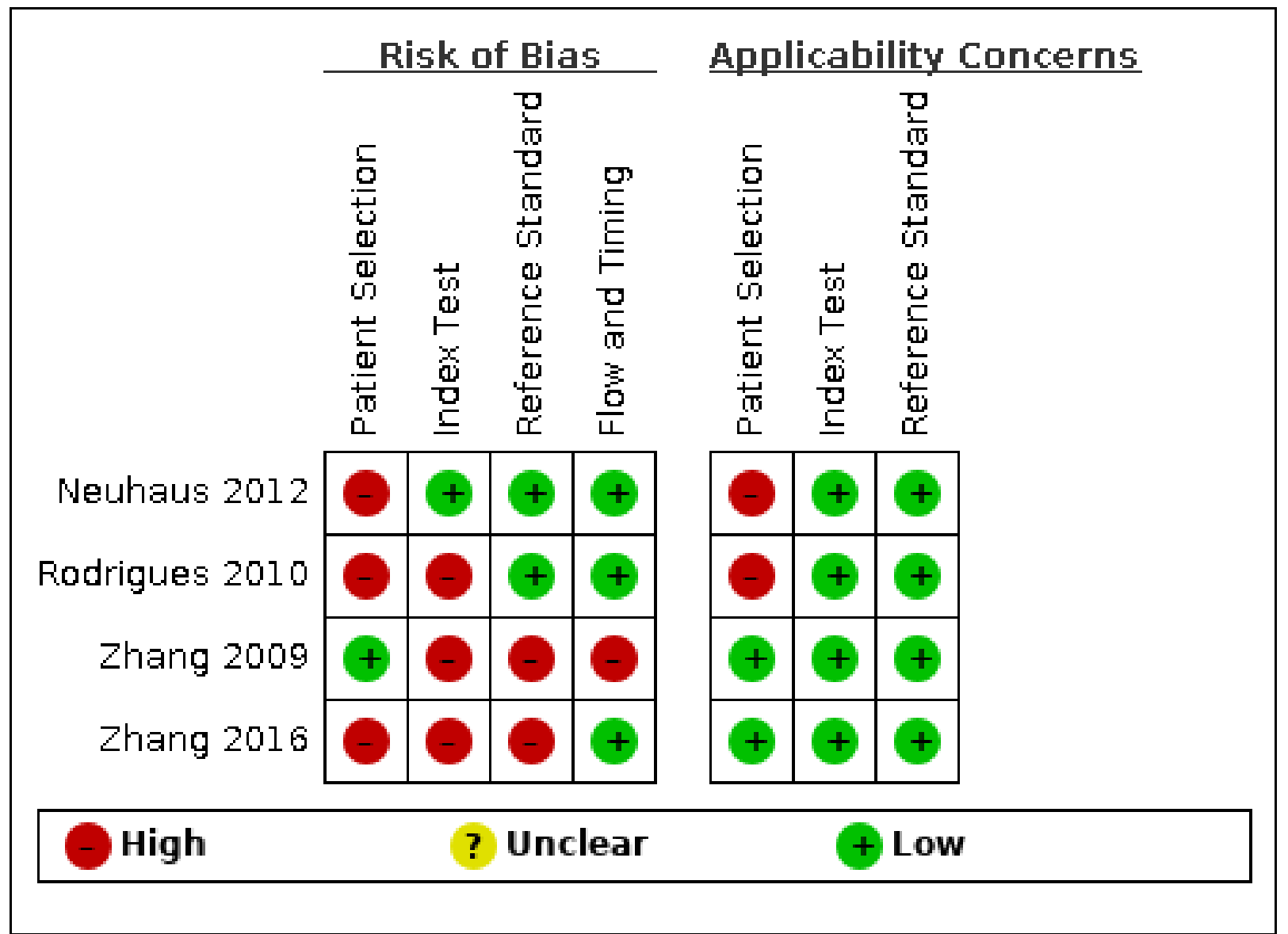

Figure 4. Risk of bias and applicability concerns graph: review authors' judgements about each domain presented as percentages across included studies.

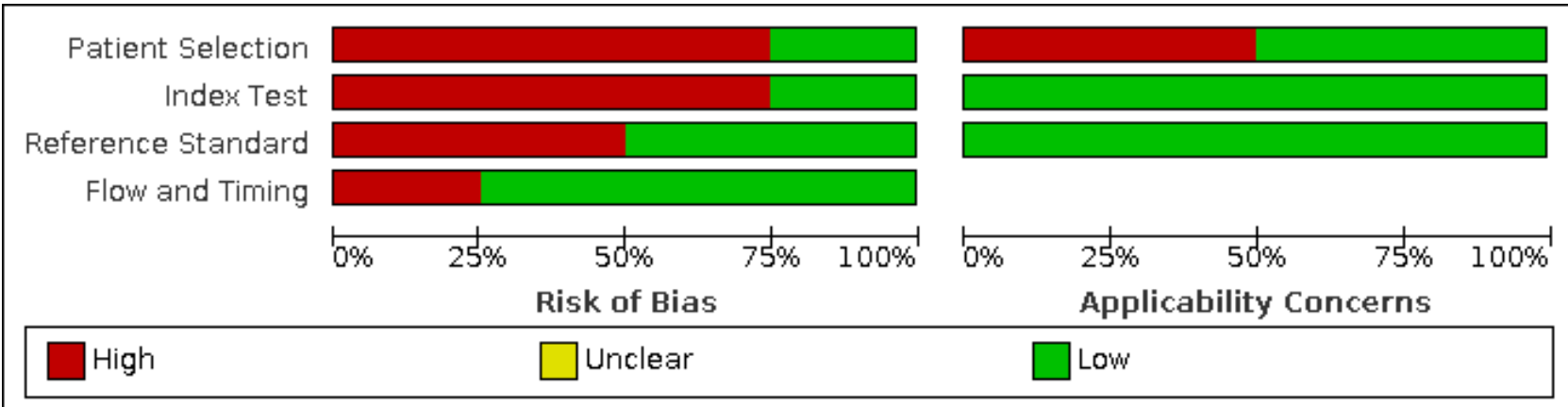

The index test was considered to be at low risk of bias in one study (Neuhaus 2012). We judged the remaining studies to be at high risk of bias as the diagnostic threshold to determine the presence of root caries was not pre-specified. Instead, the results from the threshold that conferred the highest values of sensitivity and specificity were reported. In order to minimise bias, index tests should be conducted without knowledge of the reference standard results. Two studies were conducted in vitro on extracted teeth and used histology as the reference standard, following conduction of the index tests (Neuhaus 2012; Rodrigues 2010) without knowledge of the results of the reference standard. Two studies used visual-tactile examination as the reference standard and laser fluorescence as the index test (Zhang 2009; Zhang 2016). In one study the reference standard was conducted prior to the index test and laser fluorescence assessment performed by an assessor blind to the reference standard results (Zhang 2009). In conducting laser fluorescence assessment of root surfaces it is necessary to apply the DIAGNOdent tip and therefore visualise the 
root surfaces under investigation. The plausibility of blinding a laser fluorescence assessor of the visual-tactile examination reference standard was considered. This study described a clear protocol on the conduction of DIAGNOdent including assessment of the whole root surface and recording of the maximum fluorescence reading for each surface. The assessor visualising the root surface while performing the laser fluorescence assessment was therefore considered to carry little effect on the conduction of the index test. In one study the order of index test and reference standard was unclear (Zhang 2016). Blinding of assessors was not reported and it is therefore unclear if the index test results were interpreted without knowledge of the reference standard.

Two studies used a visual-tactile examination following World Health Organization Oral Health Survey methods as the reference standard and were considered to be at high risk of bias for the reference standard (Zhang 2009; Zhang 2016). Studies that used visual-tactile examination as the reference standard may erroneously classify the target condition, particularly for noncavitated lesions with subsurface demineralisation, and these studies were judged to be at high risk of bias for the reference standard domain. Two studies (50\%) used histology as the reference standard and were considered to be at low risk of bias (Neuhaus 2012; Rodrigues 2010). As part of the risk of bias assessment, it was important that the reference standard was conducted without knowledge of the results of the index test. In three studies (75\%) the reference standard results were interpreted without knowledge of the results of the index tests.

Flow and timing was considered to be at high risk of bias in one study because 209 root surfaces $(5 \%)$ that had received both the index test and reference standard were missing from the analysis (Zhang 2009).

Two in vitro studies using previously extracted teeth (Neuhaus 2012; Rodrigues 2010) were judged as high concern for applicability of patient selection. Further, these studies investigated the accuracy of diagnostic tests in the diagnosis of secondary caries and the results may not be applicable to the diagnosis of primary caries.
All studies were judged as low concern for index test and reference standard.

\section{Findings}

We identified four studies which provided eight datasets evaluating 4997 root surfaces. All studies reported case finding (detection) rather than the consideration of patient risk and history, etc. (diagnosis). Only one study reported on the combination of index tests (Rodrigues 2010). Given the small number of studies and important differences in the clinical and methodological characteristics of the studies, meta-analysis was not considered appropriate. Consequently, we were unable to formally evaluate the comparative accuracy of the different tests considered in this review. Due to study limitations and the heterogeneity of studies and results, we judged the certainty of the evidence to be very low (Summary of findings 1 ).

Reported sensitivity and specificity estimates are presented for each of the four diagnostic tests.

\section{Laser fluorescence}

Four studies (Neuhaus 2012; Rodrigues 2010; Zhang 2009; Zhang 2016) investigated laser fluorescence and reported estimates of sensitivity ranging from 0.50 to 0.81 and estimates of specificity ranging from 0.40 to 0.80 . Two of these studies evaluated tests on in vivo primary root caries lesions using visual-tactile examination as a reference standard (Zhang 2009; Zhang 2016) (Figure 5), and two studies evaluated tests on in vitro secondary root caries lesions using histology as a reference standard (Neuhaus 2012; Rodrigues 2010) (Figure 5). Three studies investigating laser fluorescence did not pre-specify the diagnostic threshold to determine the presence of root caries and instead used the threshold at which the highest values of sensitivity and specificity were recorded (Rodrigues 2010; Zhang 2009; Zhang 2016). One study reported the optimum diagnostic threshold for secondary root caries was a fluorescence value of 18 (Rodrigues 2010). Two studies reported the optimum diagnostic threshold for primary root caries, with fluorescence values between 5 and 10 reported (Zhang 2009; Zhang 2016). 
Figure 5. Forest plot of tests: 1 Laser fluorescence, 2 Radiograph, 3 Visual examination, 4 Combined.

Laser fluorescence

$\begin{array}{lrrrrrr}\text { Study } & \text { TP } & \text { FP } & \text { FN } & \text { TN } & \text { Sensitivity }(95 \% \mathrm{Cl}) & \text { Specificity }(95 \% \mathrm{Cl}) \text { Sensitivity }(95 \% \mathrm{Cl} \text { Specificity (95\% Cl) } \\ \text { Neuhaus 2012 } & 10 & 6 & 10 & 4 & 0.50[0.27,0.73] & 0.40[0.12,0.74] \\ \text { Rodrigues 2010 } & 13 & 6 & 3 & 7 & 0.81[0.54,0.96] & 0.54[0.25,0.81] \\ \text { Zhang 2009 } & 158 & 756 & 40 & 3025 & 0.80[0.74,0.85] & 0.80[0.79,0.81] \\ \text { Zhang 2016 } & 107 & 123 & 27 & 493 & 0.80[0.72,0.86] & 0.80[0.77,0.83]\end{array}$

Radiograph

Study

Neuhaus 2012

Rodrigues 2010

TP FP FN TN Sensitivity $[95 \% \mathrm{Cl})$

$\begin{array}{lllll}8 & 2 & 12 & 8 & 0.40[0.19,0.64]\end{array}$

$0.63[0.35,0.85]$

Specificity $(95 \% \mathrm{Cl})$

$0.80[0.44,0.97]$

$0.31[0.09,0.61]$

Visual examination

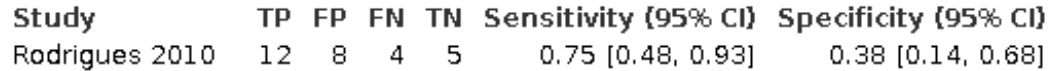

Combined

Study TP FP FN TN Sensitivity $(95 \% \mathrm{Cl})$ Specificity $[95 \% \mathrm{Cl})$

Rodrigues $2010 \quad 13 \quad 6 \quad 3 \quad 7 \quad 0.81[0.54,0.96] \quad 0.54[0.25,0.81]$
Sensitivity $(95 \%$ Cl $)$ Specificity $(95 \% \mathrm{Cl})$

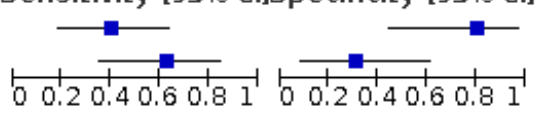

Sensitivity [95\% Cl]Specificity $[95 \% \mathrm{Cl})$

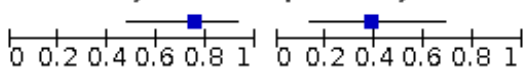

Sensitivity $[95 \%$ Cl $)$ Specificity $[95 \% \mathrm{Cl}]$

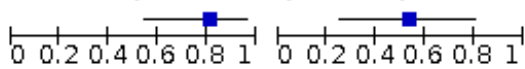

\section{Radiograph examination}

Two studies (Neuhaus 2012; Rodrigues 2010) investigated radiographic examination and reported estimates of sensitivity ranging from 0.40 to 0.63 and estimates of specificity ranging from 0.31 to 0.80 (Figure 5).

\section{Visual examination}

One study (Rodrigues 2010) investigated visual examination and reported an estimate of sensitivity of 0.75 ( $95 \%$ confidence interval (CI) 0.48 to 0.93$)$ and specificity of $0.38(95 \% \mathrm{Cl} 0.14$ to 0.68$)$ (Figure 5).

\section{Radiograph and visual examination combined}

One study (Rodrigues 2010) investigated a combined radiographic and visual examination and reported an estimate of sensitivity of $0.81(95 \% \mathrm{Cl} 0.54$ to 0.96$)$ and specificity of $0.54(95 \% \mathrm{Cl} 0.25$ to 0.81$)$. Utilising radiographic and visual examination together in this study resulted in improved sensitivity and specificity compared to the use of radiographs (sensitivity $0.63(95 \% \mathrm{Cl} 0.35$ to 0.85 , specificity 0.31 $(95 \% \mathrm{Cl} 0.09$ to 0.61$)$ or visual examination (sensitivity $0.75(95 \% \mathrm{Cl}$ 0.48 to 0.93$)$, specificity $0.38(95 \% \mathrm{Cl} 0.14$ to 0.68$)$ in isolation (Figure $5)$.

Due to the small number of included studies and the important differences in the clinical and methodological characteristics we were unable to undertake our planned investigations of heterogeneity or sensitivity analyses.

\section{DISCUSSION}

\section{Summary of main results}

The aim of this review was to estimate the accuracy of diagnostic tests for the detection of root caries lesions. The included studies evaluated four diagnostic tests - laser fluorescence, radiographs, visual examination, and a combined radiographic and visual examination.

- Four studies evaluated laser fluorescence and reported estimates of sensitivity ranging from 0.50 to 0.81 and specificity ranging from 0.40 to 0.80 .

- Two studies evaluated radiographs and reported estimates of sensitivity ranging from 0.40 to 0.63 and specificity ranging from 0.31 to 0.80 .

- One study evaluated visual examination and reported sensitivity of 0.75 (95\% confidence interval $(\mathrm{Cl}) 0.48$ to 0.93 ) and specificity of 0.38 ( $95 \% \mathrm{Cl} 0.14$ to 0.68 ).

- One study evaluated the accuracy of radiograph and visual examination in combination and reported sensitivity of 0.81 $(95 \% \mathrm{Cl} 0.54$ to 0.96$)$ and specificity of $0.54(95 \% \mathrm{Cl} 0.25$ to 0.81$)$.

Given the small number of studies and important differences in the clinical and methodological characteristics of the studies we were unable to pool the results of the studies. Consequently, we were unable to formally evaluate the comparative accuracy of the different tests considered in this review. Using the Quality Assessment of Diagnostic Accuracy Studies 2 (QUADAS-2) we judged all four studies to be at overall high risk of bias. Reasons included the bias in the selection process, use of post hoc (data driven) positivity thresholds, and the use of an imperfect reference standard.

In line with GRADE guidelines (Schünemann 2020a), we downgraded the certainty of the evidence due to study limitations and the imprecision of results (downgraded two levels), reflected in the wide confidence intervals around the sensitivity and specificity for each of the four included index tests. We therefore judged the certainty of the evidence to be very low (Summary of findings 1 ). There is no doubt that studies of caries detection and diagnosis are challenging, and some of the study limitations are inherent in the 
nature of the disease under study. Other study limitations observed in the included studies are surmountable.

The patient selection and index test domains were most problematic in terms of bias. Few studies employed consecutive or random selection and often the methods used were not clearly reported. The use of consecutive or random selection minimises the likelihood that teeth are explicitly selected for inclusion which may be either simpler or more complex to diagnose. Applicability of patient selection was also of concern for two in vitro studies. In vitro testing is an important part of the development of diagnostic tests, however these studies inevitably cause high concern for applicability to our research question which aimed to determine the accuracy of these devices when used in a clinical setting which poses particular challenges in terms of difficulty of access to the oral cavity, presence of plaque and saliva, patient acceptability, and time constraints for examination. Furthermore, the focus of these studies was secondary root caries lesions and the results may not be applicable to primary root caries lesions the target condition of this review.

The lack of a pre-specified threshold at which to determine the presence of root caries was a limitation in three studies evaluating laser fluorescence. The order of assessment of index test and reference standard, and the blinding of assessors was not always clearly stated.

Two in vivo studies used a visual-tactile examination as the reference standard and whilst it is difficult to conceive of another reference standard suitable for use at this present time for disease-free participants, uncertainty remains around anything other than a comprehensive, enhanced visual assessment such as the International Caries Detection and Assessment System (ICDAS) to correctly classify root caries lesions. Whilst histology is the preferred reference standard this is not appropriate for use in in vivo studies. This highlights a key difficulty in studies of diagnostic test accuracy: in order to correctly classify the target condition histology is the most appropriate reference standard but if extracted teeth (in vitro studies) are selected for inclusion then this reduces generalizability of the results and limited applicability. One potential solution is to recruit patients where a tooth extraction is indicated. In such circumstances an index test can be applied in vivo and the histology reference standard applied following extraction. Such studies can be logistically difficult to design and conduct and depending on the population recruited may still cause concern if, for example, the study sample is composed of adolescents requiring extractions for orthodontic purposes who could potentially have a lower prevalence of caries than adults. Designing a study like this may be even more challenging where the target condition is root caries, where affected patients are older (Domejean 2017; White 2012) and therefore teeth are not often indicated for extraction. Adult populations may undergo tooth loss as a result of periodontal disease but challenges in organising and administering these studies would remain, along with issues regarding applicability considering the reported significant association between gingival recession, bleeding on probing, and root caries (Sugihara 2010). Equally, designing a study investigating the target condition of primary root caries presents challenges given that older populations are affected more commonly by root caries and also have higher mean numbers of restored teeth compared to younger populations (White 2012). The decision to include secondary root caries within this review was made due to the small number of available studies investigating primary root caries, perhaps as a result of the challenges in designing a study in older populations with carious root surfaces in otherwise unrestored teeth.

When clinicians encounter patients with exposed root surfaces the initial assessment of the root surface is carried out based on its visual appearance and tactile sensation. There are limitations to a visual and tactile examination, with conflicting views on the diagnostic value of lesion colour with respect to its activity (Carvalho 2017). The aim of this review was to assess the accuracy of diagnostic tests in the diagnosis of root caries and to that end one study tested the accuracy of visual examination (Rodrigues 2010), albeit investigation of approximal secondary caries. This study reported a sensitivity of $0.75(95 \% \mathrm{Cl} 0.48$ to 0.93$)$ and specificity 0.38 ( $95 \% \mathrm{Cl} 0.14$ to 0.68$)$, representing a high number of false positives when using visual examination in isolation. This was the only study that investigated the accuracy of index tests in combination and found that utilising radiographic examination in combination with a visual examination resulted in improved sensitivity of $0.81(95 \% \mathrm{Cl} 0.54$ to 0.96$)$ and specificity of 0.54 (95\% $\mathrm{Cl} 0.25$ to 0.81 ) compared to a visual examination in isolation. There were no studies investigating the accuracy of visual examination for detecting primary caries. This review attempted to investigate whether other technologies may be recommended for use in the diagnosis of root caries in addition to the conventional oral examination as part of a routine clinical examination, however given the small number of included studies many uncertainties remain. Although addition of the radiographic examination to the visual exam improved specificity, false positives are still a major problem with nearly $50 \%$ of sound roots being incorrectly diagnosed as carious. This may be a problem associated with cervical burnout in the approximal root surfaces on radiograph.

In all four studies root surfaces underwent removal of calculus with a dental scaler prior to index tests or reference standard. In addition to investigating the accuracy of laser fluorescence in the detection of root caries lesions, one study also investigated the effect of dental scaling on detection of root caries by laser fluorescence (Zhang 2016). This study found that calculus significantly affected the accuracy of laser fluorescence, with all DIAGNOdent readings obtained from sound root surfaces with calculus returning readings of greater than 50, which might be misinterpreted as caries. The presence of plaque on root surfaces also resulted in slightly higher DIAGNOdent readings compared to sound root surfaces after dental scaling, with the sensitivity and specificity of DIAGNOdent improving following scaling. DIAGNOdent and pen work on the principle that bacterial byproducts (porphyrins) fluoresce under the appropriate wavelength light and hence when plaque and calculus are present fluorescence results are disproportionately high, and so use of DIAGNOdent may only be recommended on root surfaces without plaque or calculus.

The texture of a root caries lesion on tactile examination has been considered of diagnostic value with respect to lesion activity. Arrested lesions have been closely associated with a hard texture on gentle probing and active lesions closely associated with a soft or leathery texture on probing (Carvalho 2017; Ekstrand 2013). Zhang 2009 and Zhang 2016 included lesion texture within their clinical criteria for defining the caries status of root surfaces by visualtactile examination. Both studies reported significantly different DIAGNOdent readings from active root caries lesions and inactive 
lesions where root surfaces where plaque-free. There is therefore a suggestion that laser fluorescence may have a role in longitudinal monitoring of root caries lesion activity.

Fifteen studies published between 1985 and 2018 were excluded from this review: four studies were not diagnostic test accuracy (DTA) studies, four studies contained no sound teeth, three studies had no reference standard, two studies did not report root caries data in a way that allowed construction of a $2 \times 2$ table, and two studies included artificially created caries lesions. Of the four studies excluded for not incorporating DTA methods, one study investigated index tests laser fluorescence and visualtactile examination (Karlsson 2009), one study investigated thermal and near-infrared (NIR) reflectance imaging and polarization sensitive optical coherence tomography (PS-OCT) (Lee 2017), one investigated electronic caries monitor (ECM III) and laser fluorescence (Wicht 2002), and one investigated swept-source optical coherence tomography (Zhou 2018). Of the two studies where root caries data were not reported in a way that allowed construction of a $2 \times 2$ table, both investigated the diagnostic accuracy of radiographic examination (Nordenram 1988; Sikri 1991).

Electronic caries monitor (ECM) is based on the measurement of electrical resistance of dental tissues during controlled drying. The electrical resistance of enamel and dentine increases as remineralisation of the tissue surface and subsequent decrease in pore volume occurs (Petersson 2004). Baysan reported increased electrical resistance of root caries lesions treated with 5000 parts per million (ppm) fluoride dentifrice compared to lesions treated with $1100 \mathrm{ppm}$ fluoride dentifrice over a six-month period (Baysan 2001). These studies provide evidence that remineralisation of root caries lesions can be related to electrical resistance measurements and suggest a role for ECM in longitudinal monitoring of root caries lesions (Pretty 2013; Pretty 2017).There is evidence that ECM and infrared imaging modalities may be suitable methods of diagnosing and monitoring root caries lesions however, we were unable to include studies in this review where DTA methods were not incorporated.

\section{Strengths and weaknesses of the review}

The strengths of this Cochrane Review are the completion of multiple, comprehensive, electronic literature searches, rigorous application of methodology which ensured that all screening, inclusion decisions, and data extraction and quality assessments were performed in duplicate by a multidisciplinary team of clinicians and methodologists. A clear and reproducible method was utilised for the application of methodological quality decisions.

This is the first diagnostic test accuracy review to report the detection of root caries lesions separately from coronal caries lesions. A systematic review by Brouwer investigated the accuracy of diagnostic tests in the detection of secondary caries lesions (Brouwer 2016). This review included 23 studies with two studies providing data for root caries lesions. The data for root caries were combined with data provided for occlusal caries within the summary receiver operating characteristic (SROC) curves and analysed as non-proximal lesions to compare with the sensitivity and specificity of diagnostic tests for proximal lesions. Given the notable differences in the histopathology of root caries and coronal caries, and diagnostic specificities of root caries (Damé-Teixeira
2017), it was decided the analysis of the accuracy of diagnostic tests for root caries lesions independent of coronal lesions was more appropriate for our review and would be relevant to clinical practice.

The main weakness of this review is the low number of included studies, reflecting the sparse evidence base in this area, and also poor reporting of primary studies where data were presented in a format that did not permit construction of a $2 \times 2$ table or where DTA methodology was not employed in the design or conduct of the study or both. Four studies were ineligible for inclusion as they were not reported as DTA studies and two studies were excluded because they did not report data in a way that allowed construction of a 2 $\times 2$ table.

A final area of concern was the effect of the chosen threshold of included root caries lesions. Early detection of root caries lesions is essential if the effect of preventive interventions is to be maximised. Studies investigating the arrest of root caries lesions have reported a higher rate of remineralisation in noncavitated lesions than in cavitated lesions (Baysan 2001; DePaola 1993; Ekstrand 2013). The lower arrest rate of cavitated lesions may indicate that the dentine is more demineralised and less able to provide a suitable substrate for remineralisation (Heilman 1997; Wefel 1995). Alternatively, the arrest rate may be affected by difficulties in maintaining effective plaque control in cavitated lesions.

For this reason, the detection of root caries lesions prior to lesion cavitation is considered important. However, the inclusion or exclusion of either cavitated or non-cavitated root caries lesions and their effect on the diagnostic test accuracy of any of the included index tests is not reported in any of the studies included in this review.

\section{Applicability of findings to the review question}

There are some concerns regarding the clinical applicability of the findings of this review based on the inclusion of two studies assessing the detection of secondary root caries lesions in vitro, conducted in a non-clinical setting which is unrepresentative of the general dental setting.

There are also some concerns regarding the conduct of the index test in the two in vivo studies. The diagnostic threshold of the laser fluorescence index tests to determine the presence of root caries was not pre-specified. Instead, the results from the threshold that conferred the highest values of sensitivity and specificity were reported, potentially overestimating the accuracy of the index tests. To improve clinical applicability the diagnostic thresholds of diagnostic tests should be pre-specified and ideally utilise the thresholds detailed by manufacturers.

\section{AUTHORS'CONCLUSIONS}

\section{Implications for practice}

This Cochrane Review was undertaken to provide results that are directly applicable to general dental practitioners. Ideally clinicians would have all diagnostic tests available to them and use the most appropriate tests indicated by the clinical scenario. As part of a dental examination clinicians will always assess dental surfaces visually, but then consider adjunctive use of diagnostic tests that could provide additional signs to inform diagnosis. 
In consideration of the supplementary use of diagnostic tests, clinicians will consider the risks or harms of these investigations against the likely benefits of additional information. The benefits of additional information from supplementary diagnostic tests is likely to change depending on the certainty of the diagnosis based on visual-tactile examination alone, which is likely to change according to the clinical scenario. Visual-tactile examination is the mainstay of root caries diagnosis; however, due to the paucity of the evidence base and the very low certainty of evidence the additional benefit of adjunctive diagnostic tests cannot be established by this review.

\section{Implications for research}

There was a small number of studies included in this review and due to important differences in the clinical and methodological characteristics of the included studies, meta-analysis was not appropriate. Additional diagnostic test accuracy studies addressing this area of research are warranted. Given the number of studies excluded from this review which did not report data in a useable format, it is of vital importance that future diagnostic studies report results in a clear method, following the STARD checklist (Bossuyt 2015), ideally with a cross-tabulation of the index test and reference standard to allow construction of a $2 \times 2$ table to allow extraction of true-positive, false-positive, false-negative, and true-negative results.

Future studies should be aware of the importance of sampling participants using consecutive recruitment or random sampling to minimise the introduction of bias from the selection of teeth in which the detection of root caries lesions is either more straightforward or more difficult. Studies should also specify index test thresholds a priori rather than utilising a threshold which maximises test sensitivity and specificity. The use of manufacturer recommended thresholds or those validated in previous research studies are recommended. Studies may be conducted with the objective of identifying the most accurate diagnostic threshold for a specific population. We would recommend that the manufacturers recommended thresholds are reported in addition to the optimum thresholds to facilitate comparison between the two and allow for analysis in future reviews. Future studies should use a reference standard that can correctly classify the target condition.

Three studies in this review used visual or visual-tactile examination of root caries lesions as either an index test or reference standard (Rodrigues 2010; Zhang 2009; Zhang 2016). Two studies reported the clinical criteria used to define root caries using visual-tactile examination and one did not. A significant challenge in research in this field is the lack of consensus on a definition for root caries, with diagnostic criteria differing between studies (Hayes 2017). The current level of clinical heterogeneity between studies in this area makes meta-synthesis of results difficult and in some cases even inappropriate. The call for agreement in this area is not a new one (Banting 1986; Ritter 2010), however a consensus on the clinical criteria used to define root caries is needed to promote consistent reporting between studies and allow metasynthesis of their results.

Studies where non-caries related tooth extraction is indicated will enable the index test to be conducted in vivo and a reference standard of histological assessment following extraction. Given the importance of initiating non-operative interventions to arrest active root caries lesions prior to lesion cavitation, inclusion and accurate reporting of non-cavitated root caries lesions in future studies is essential to assess the accuracy of diagnostic tests at this threshold.

Randomised controlled trials could be beneficial by investigating the longer-term effects of tests for detection, diagnosis, and monitoring of root caries lesions on disease prevention through active preventive interventions.

\section{ACKNOWLEDGEMENTS}

This series of Cochrane Reviews was funded by the UK National Institute for Health Research (NIHR) Cochrane Programme Grant Scheme (Project: 16/114/23). We would like to thank Anne Littlewood (Information Specialist, Cochrane Oral Health) for her advice on the search strategy and conducting the search of the literature, and Luisa M Fernandez Mauleffinch (Managing Editor and Copy Editor, Cochrane Oral Health) for her assistance in facilitating this review. We thank Associate Professor KR Ekstrand, J Bader (Emeritus Professor, UNC School of Dentistry, Chapel Hill North Carolina, USA), lain Pretty, and Louise White for their feedback on the protocol; and Jennifer Hilgart, Rodrigo A Giacaman (Cariology Unit, Department of Oral Rehabilitation, Faculty of Health Sciences, University of Talca, Talca, Chile), Edward CM Lo (Faculty of Dentistry, University of Hong Kong), and the Cochrane Diagnostic Test Accuracy Editorial Team for their feedback on the review. Also Alex Sutton and Suzanne Freeman from the NIHR Complex Review Support Unit for their support on this review. 


\section{REFERE N CES}

\section{References to studies included in this review}

Neuhaus 2012 \{published data only\}

Neuhaus KW, Rodrigues JA, Seemann R, Lussi A. Detection of proximal secondary caries at cervical class IIamalgam restoration margins in vitro. Journal of Dentistry 2012;40(6):493-9.

\section{Rodrigues 2010 \{published data only\}}

Rodrigues JA, Neuhaus KW, Hug I, Stich H, Seemann R, Lussi A. In vitro detection of secondary caries associated with composite restorations on approximal surfaces using laser fluorescence. Operative Dentistry 2010;35(5):564-71.

\section{Zhang 2009 \{published data only\}}

Zhang W, McGrath C, Lo EC. A comparison of root caries diagnosis based on visual-tactile criteria and DIAGNOdent in vivo. Journal of Dentistry 2009;37(7):509-13.

\section{Zhang 2016 \{published data only\}}

Zhang W, McGrath C, Lo EC. Effectiveness of DIAGNOdent in detecting root caries without dental scaling among community-dwelling elderly. Oral Health \& Preventive Dentistry 2016;14(6):555-61.

\section{References to studies excluded from this review}

Amaechi 2004 \{published data only\}

Amaechi BT, Podoleanu AG, Komarov G, Higham SM, Jackson DA. Quantification of root caries using optical coherence tomography and microradiography: a correlational study. Oral Health \& Preventive Dentistry 2004;2(4):377-82.

\section{Baysan 2004 \{published data only\}}

Baysan A, Prinz JF, Lynch E. Clinical criteria used to detect primary root caries with electrical and mechanical measurements in vitro. American Journal of Dentistry 2004;17(2):94-8

\section{Darling 2012 \{published data only\}}

Darling CL, Staninec M, Chan KH, Kang H, Fried D. Remineralization of root caries monitored using cross polarization optical coherence tomography. Proceedings of SPIE - the International Society for Optical Engineering 2012;8208:82080V. [DOI: 10.1117/12.914633]

\section{Ekstrand 2007 \{published data only\}}

Ekstrand KR, Martignon S, Ricketts DJ, Qvist V. Detection and activity assessment of primary coronal caries lesions: a methodologic study. Operative Dentistry 2007;32(3):225-35.

\section{Fried 2002 \{published data only\}}

Fried D, Xie J, Shafi S, Featherstone JDB, Breunig TM, Le C. Imaging caries lesions and lesion progression with polarization sensitive optical coherence tomography. Journal of Biomedical Optics 2002;7:618-27.
Galal 1985 \{published data only\}

Galal A, Manson-Hing L, Jamison H. A comparison of combinations of clinical and radiographic examinations in evaluation of a dental clinic population. Oral Surgery, Oral Medicine, and Oral Pathology 1985;60(5):553-61.

\section{Karlsson 2009 \{published data only\}}

Karlsson L, Johansson E, Tranaeus S. Validity and reliability of laser-induced fluorescence measurements on carious root surfaces in vitro. Caries Research 2009;43(5):397-404.

Lee 2017 \{published data only\}

Lee RC, Darling CL, Staninec M, Ragadio A, Fried D. Activity assessment of root caries lesions with thermal and near-IR imaging methods. Journal of Biophotonics 2017;10(3):433-45.

Lynch 1994 \{published data only\}

Lynch E, Beighton D. A comparison of primary root caries lesions classified according to colour. Caries Research 1994;28(4):233-9.

\section{Mello 2011 \{published data only\}}

Mello TRC, Sousa MLR, Antunes JLF, Meirelles MPMR, Ellwood RP. Early diagnosis of root caries in dental public health. American Journal of Epidemiology 2011;173(Suppl_11):S325 (Abs No L33).

\section{Nordenram 1988 \{published data only\}}

Nordenram G, Bergkvist A, Johnson G, Henriksson CO, Anneroth G. Macroscopic and radiographic examination of proximal root surface caries. Acta Odontologica Scandinavica 1988;46(2):95-9.

\section{Ratanaporncharoen 2018 \{published data only\}}

Ratanaporncharoen C, Tabata M, Kitasako Y, Ikeda M, Goda T, Matsumoto $\mathrm{A}$, et al. $\mathrm{pH}$ mapping on tooth surfaces for quantitative caries diagnosis using micro $\mathrm{Ir} / \mathrm{lrOx} \mathrm{pH}$ sensor. Analytical Chemistry 2018;90(7):4925-31.

\section{Sikri 1991 \{published data only\}}

Sikri V, Sikri P. Clinical and radiological examination of root surface caries. An in vitro study. Indian Journal of Dental Research 1991;2(1-2):1-6.

Wicht 2002 \{published data only\}

Wicht MJ, Haak R, Stutzer H, Strohe D, Noack MJ. Intra- and interexaminer variability and validity of laser fluorescence and electrical resistance readings on root surface lesions. Caries Research 2002;36(4):241-8.

\section{Zhou 2018 \{published data only\}}

Zhou Y, Shimada Y, Matin K, Sadr A, Yoshiyama M, Sumi Y, et al. Assessment of root caries under wet and dry conditions using swept-source optical coherence tomography (SS-OCT). Dental Materials Journal 2018;37(6):880-8. 


\section{Additional references}

\section{Adult Dental Health Survey 2009}

NHS Digital 2011. Adult Dental Health Survey 2009 - Summary report and thematic series. digital.nhs.uk/data-andinformation/publications/statistical/adult-dental-healthsurvey/adult-dental-health-survey-2009-summary-report-andthematic-series\#resources (accessed 2 August 2018).

\section{Anaise 1984}

Anaise JZ. Measurement of dental caries experience modification of the DMFT index. Community Dentistry and Oral Epidemiology 1984;12(1):43-6.

\section{Angmar-Månsson 2001}

Angmar-Månsson B, Ten Bosch JJ. Quantitative light-induced fluorescence (QLF): a method for assessment of incipient caries lesions. Dento Maxillo Facial Radiology 2001;30:298-307.

\section{Atkins 2004}

Atkins D, Eccles M, Flottorp S, Guyatt GH, Henry D, Hill S, et al. Systems for grading the quality of evidence and the strength of recommendations I: critical appraisal of existing approaches The GRADE Working Group. BMC Health Services Research 2004;4(1):38.

\section{Banting 1986}

Banting DW. Epidemiology of root caries. Gerodontology 1986;5:5-11.

\section{Banting 2001}

Banting DW. The diagnosis of root caries. Journal of Dental Education 2001;65(10):991-6.

\section{Baysan 2001}

Baysan A, Lynch E, Ellwood R, Davies R, Petersson L, Borsboom P. Reversal of primary root caries using dentifrices containing 5000 and 1100 ppm fluoride. Caries Research 2001;35(1):41-6

\section{Bossuyt 2015}

Bossuyt PM, Reitsma JB, Bruns DE, Gatsonis CA, Glasziou PP, Irwig L, et al. STARD 2015: an updated list of essential items for reporting diagnostic accuracy studies. Clinical Chemistry 2015;61(12):1446-52.

\section{Braga 2009}

Braga MM, Oliveira LB, Bonini GAVC, Bönecker M, Mendes FM. Feasibility of the International Caries Detection and Assessment System (ICDAS-II) in epidemiological surveys and comparability with standard World Health Organization criteria. Caries Research 2009;43:245-9.

\section{Brouwer 2016}

Brouwer F, Askar H, Paris S, Schwendicke F. Detecting secondary caries lesions: a systematic review and metaanalysis. Journal of Dental Research 2016;95(2):143-51.

\section{Burrow 2017}

Burrow MF, Stacey MA. Management of cavitated root caries lesions: minimum intervention and alternatives. In: Carrilho
MRO, editors(s). Root Caries: from Prevalence to Therapy. Vol. 26. Basel: Karger, 2017:106-14.

\section{Carvalho 2017}

Carvalho TS, Lussi A. Assessment of root caries lesion activity and its histopathological features. In: Carrilho MRO, editors(s). Root Caries: from Prevalence to Therapy. Vol. 26. Basel: Karger, 2017:63-9.

\section{Christensen 2015}

Christensen L, Bardow A, Ekstrand K, Fiehn N, Heitmann B, Qvist V, et al. Root caries, root surface restorations and lifestyle factors in adult Danes. Acta Odontologica Scandinavica 2015;73:467-73.

\section{Damé-Teixeira 2017}

Damé-Teixeira N, Parolo CCF, Maltz M. Specificities of caries on root surface. In: Carrilho MRO, editors(s). Root Caries: from Prevalence to Therapy. Vol. 26. Basel: Karger, 2017:15-25.

Davies 2001

Davies GM, Worthington HV, Clarkson JE, Thomas P, Davies RM. The use of fibre-optic transillumination in general dental practice. British Dental Journal 2001;191(3):145-7.

\section{Deeks 2005}

Deeks JJ, Macaskill P, Irwig L. The performance of tests of publication bias and other sample size effects in systematic reviews of diagnostic test accuracy was assessed. Journal of Clinical Epidemiology 2005;58(9):882-93.

\section{DePaola 1993}

DePaola PF. Caries in our aging population: what are we learning? In: Bowen WH, Tabak LA, editors(s). Cariology for the Nineties. Rochester: University of Rochester Press, 1993:25-35.

\section{Domejean 2017}

Domejean S, Banerjee A. Assessing the risk of developing carious lesions in root surfaces. In: Carrilho MRO, editors(s). Root Caries: from Prevalence to Therapy. Vol. 26. Basel: Karger, 2017:55-62.

\section{Ekstrand 2008}

Ekstrand K, Martignon S, Holm-Pedersen P. Development and evaluation of two root caries controlling programmes for home-based frail people older than 75 years. Gerodontology 2008;25:67-75

\section{Ekstrand 2013}

Ekstrand KR, Poulsen JE, Hede B, Twetman S, Qvist V, Ellwood RP. A Randomized Clinical Trial of the Anti-Caries Efficacy of 5,000 Compared to 1,450 ppm Fluoridated Toothpaste on Root Caries Lesions in Elderly Disabled Nursing Home Residents. Caries Research 2013;47:391-398.

\section{Elderton 2003}

Elderton RJ. Preventive (evidence-based) approach to quality general dental care. Medical Principles and Practice 2003;12 Suppl 1:12-21. 


\section{Ellwood 1997}

Ellwood RP, Davies RM, Worthington HV. Evaluation of a dental subtraction radiography system. Journal of Periodontal Research 1997;32:241-8.

\section{Fejerskov 1991}

Fejerskov O, Luan WM, Nyvad B, Budtz-Jorgensen E, HolmPedersen. Active and inactive root surface caries lesions in a selected group of 60- to 80-year-old Danes. Caries Research 1991;25(5):385-91.

\section{Fejerskov 2015}

Fejerskov O, Nyvad B, Kidd E, editor(s). Dental Caries: the Disease and its Clinical Management. 3rd edition. WileyBlackwell, 2015.

\section{Fure 1997}

Fure S, Zickert I. Incidence of tooth loss and dental caries in 60-, 70- and 80-year-old Swedish individuals. Community Dentistry and Oral Epidemiology 1997;25:137-42.

\section{Hayes 2016}

Hayes M, Brady P, Burke FM, Allen PF. Failure rates of class V restorations in the management of root caries in adults - a systematic review. Gerodontology 2016;33(3):299-307.

\section{Hayes 2017}

Hayes M, Burke F, Allen PF. Incidence, prevalence and global distribution of root caries. In: Carrilho MRO, editors(s). Root Caries: from Prevalence to Therapy. Vol. 26. Basel: Karger, 2017:1-8.

\section{Heilman 1997}

Heilman JR, Jordan TH, Warwick R, Wefel JS. Remineralization of root surfaces demineralized in solutions of differing fluoride levels. Caries Research 1997;31:423-8.

\section{Horner 2009}

Horner K, Islam M, Flygare L, Tsiklakis K, Whaites E. Basic principles for use of dental cone beam computed tomography: consensus guidelines of the European Academy of Dental and Maxillofacial Radiology. Dento Maxillo Facial Radiology 2009;38:187-95.

\section{Hsu 2011}

Hsu J, Brożek JL, Terracciano L, Kreis J, Compalati E, Stein AT, et al. Application of GRADE: making evidence-based recommendations about diagnostic tests in clinical practice guidelines. Implementation Science 2011;6(1):62.

\section{Ismail 2015}

Ismail AI, Pitts NB, Tellez M. The International Caries Classification and Management System $\left(\right.$ ICCMS $\left.^{\mathrm{TM}}\right)$ an example of a caries management pathway. BMC Oral Health 2015;15 Suppl 1:S9. [DOI: 10.1186/1472-6831-15-S1-S9]

\section{Jones 2017}

Jones CM, Davies GM, Monaghan N, Morgan MZ, Neville JS, Pitts NB. The caries experience of 5 year-old children in Scotland in 2013-2014, and in England and Wales in 2014-2015.
Reports of cross-sectional dental surveys using BASCD criteria. Community Dental Health 2017;34:157-62.

\section{Kassebaum 2015}

Kassebaum NJ, Bernabé E, Dahiya M, Bhandari B, Murray CJL, Marcenes W. Global burden of untreated caries: a systematic review and metaregression. Journal of Dental Research 2015;94:650-8

\section{Katz 1982}

Katz RV, Hazen SP, Chilton NW, Mumma RD. Prevalence and intraoral distribution of root caries in an adult population. Caries Research 1982;16:265-71.

\section{Kidd 2004}

Kidd EAM, Fejerskov O. What constitutes dental caries? Histopathology of carious enamel and dentin related to the action of cariogenic biofilms. Journal of Dental Research 2004:83:35-8.

\section{Kidd 2016}

Kidd EAM, Fejerskov O. Essentials of Dental Caries. 4th edition. Oxford University Press, 2016.

\section{Leeflang 2008}

Leeflang MMG, Deeks JJ, Gatsonis C, Bossuyt PMM. Systematic reviews of diagnostic test accuracy. Annals of Internal Medicine 2008;149:889-97.

\section{Macaskill 2010}

Macaskill P, Gatsonis C, Deeks JJ, Harbord RM, Takwoingi Y. Chapter 10: Analysing and presenting results. In: Deeks JJ, Bossuyt PM, Gatsonis C, editor(s). Cochrane Handbook for Systematic Reviews of Diagnostic Test Accuracy Version 1.0. The Cochrane Collaboration, 2010. Available from srdta.cochrane.org.

\section{Macey 2018}

Macey R, Walsh T, Riley P, Glenny A-M, Worthington HV, Clarkson JE, et al. Tests to detect and inform the diagnosis of caries. Cochrane Database of Systematic Reviews 2018, Issue 12. Art. No: CD013215. [DOI: 10.1002/14651858.CD013215]

\section{Maltz 2017}

Maltz M, Alves LS, Zenkner JEA. Biofilm control and oral hygiene practices. In: Carrilho MRO, editors(s). Root Caries: from Prevalence to Therapy. Vol. 26. Basel: Karger, 2017:76-82.

\section{Martignon 2019}

Martignon S, Pitts NB, Goffin G, Mazevet M, Douglas GVA, Newton JT, et al. CariesCare practice guide: consensus on evidence into practice. British Dental Journal 2019;227(5):353-62.

\section{Matos 2011}

Matos R, Novaes TF, Braga MM, Siqueira WL, Duarte DA, Mendes FM. Clinical performance of two fluorescence-based methods in detecting occlusal caries lesions in primary teeth. Caries Research 2011;45:294-302. 


\section{McInnes 2018}

McInnes MDF, Moher D, Thombs BD, McGrath TA, Bossuyt PM, Clifford T, et al. Preferred Reporting Items for a Systematic Review and Meta-analysis of Diagnostic Test Accuracy Studies: the PRISMA-DTA Statement. JAMA 2018;319:388-96.

\section{Meyer-Lueckel 2019}

Meyer-Lueckel H, Machiulskiene V, Giacaman RA. How to intervene in the root caries process? Systematic review and meta-analyses. Caries Research 2019;53:599-608.

\section{Muller 2007}

Muller F, Schimmel M. Management of preventative care for an ageing population. International Dental Journal 2007;57(S3):215-20.

\section{Petersen 2005}

Petersen PE, Bourgeois D, Ogawa H, Estupinan-Day S, Ndiaye C. The global burden of oral diseases and risks to oral health. Bulletin of the World Health Organization 2005;83:661-9.

\section{Petersen 2008}

Petersen PE. World Health Organization global policy for improvement of oral health - World Health Assembly 2007. International Dental Journal 2008;58(3):115-21.

\section{Petersson 2004}

Petersson LG, Kambara M. Remineralisation study of artificial root caries lesions after fluoride treatment. An in vitro study using electric caries monitor and transversal micro-radiography. Gerodontology 2004;21:85-92.

\section{Pitts 1988}

Pitts NB, Fyffe HE. The effect of varying diagnostic thresholds upon clinical caries data for a low prevalence group. Journal of Dental Research 1988;67:592-6.

\section{Pitts 2001}

Pitts NB. Clinical diagnosis of dental caries: a European perspective. Journal of Dental Education 2001;65:972-8.

\section{Pitts 2009}

Pitts N. Detection, Assessment, Diagnosis and Monitoring of Caries. Basel: Karger, 2009.

\section{Pitts 2017}

Pitts NB, Zero DT, Marsh PD, Ekstrand K, Weintraub JA, RamosGomez F, et al. Dental caries. Nature Reviews Disease Primers 2017;3:17030.

\section{Pretty 2006}

Pretty IA. Caries detection and diagnosis: novel technologies. Journal of Dentistry 2006;34:727-39.

\section{Pretty 2013}

Pretty IA, Ellwood RP. The caries continuum: opportunities to detect, treat and monitor the re-mineralization of early caries lesions. Journal of Dentistry 2013;41(Suppl 2):S12-S21.

\section{Pretty 2017}

Pretty IA. Monitoring of root caries lesions. In: Carrilho MRO, editors(s). Root Caries: from Prevalence to Therapy. Vol. 26. Basel: Karger, 2017:70-5.

\section{Reitsma 2005}

Reitsma JB, Glas AS, Rutjes AWS, Scholten RJPM, Bossuyt PM, Zwinderman AH. Bivariate analysis of sensitivity and specificity produces informative summary measures in diagnostic reviews. Journal of Clinical Epidemiology 2005;58:982-90.

\section{Review Manager 2020 [Computer program]}

Nordic Cochrane Centre, The Cochrane Collaboration Review Manager 5 (RevMan 5). Version 5.4. Copenhagen: Nordic Cochrane Centre, The Cochrane Collaboration, 2020.

\section{Ritter 2010}

Ritter AV, Shugars DA, Bader JD. Root caries risk indicators: a systematic review of risk models. Community Dentistry and Oral Epidemiology 2010;38:383-97.

\section{Rodrigues 2011}

Rodrigues JA, Lussi A, Seemann R, Neuhaus KW. Prevention of crown and root caries in adults. Periodontology 2000 2011;55(1):231-49.

\section{Rutter 2001}

Rutter CM, Gatsonis CA. A hierarchical regression approach to meta-analysis of diagnostic test accuracy evaluations. Statistics in Medicine 2001;20(19):2865-84.

\section{Schünemann 2020}

Schünemann HJ, Mustafa RA, Brozek J, Steingart KR, Leeflang M, Murad MH, et al. GRADE guidelines: 21 part 1 . Study design, risk of bias, and indirectness in rating the certainty across a body of evidence for test accuracy. Journal of Clinical Epidemiology 2020;122:129-41.

\section{Schünemann 2020a}

Schünemann HJ, Mustafa RA, Brozek J, Steingart KR, Leeflang M, Murad MH, et al. GRADE guidelines: 21 part 2. Test accuracy: inconsistency, imprecision, publication bias, and other domains for rating the certainty of evidence and presenting it in evidence profiles and summary of findings tables. Journal of Clinical Epidemiology 2020;122:142-52.

\section{Schwendicke 2018}

Schwendicke F, Krois J, Schiffner U, Wolfgang M, Rainer JA. Root caries experience in Germany 1997 to 2014: analysis of trends and identification of risk factors. Journal of Dentistry 2018;78:100-5.

\section{Shoaib 2009}

Shoaib L, Deery CM, Ricketts DNJ, Nugent ZJ. Validity and reproducibility of ICDAS II in primary teeth. Caries Research 2009;43:442-8.

\section{Slade 1996a}

Slade GD, Spencer AJ, Locker D, Hunt RJ, Strauss RP, Beck JD. Variations in the social impact of oral conditions among older 
adults in South Australia, Ontario, and North Carolina. Journal of Dental Research 1996;75:1439-50.

\section{Slade 1996b}

Slade GD, Spencer AJ, Roberts-Thomson K. Tooth loss and chewing capacity among older adults in Adelaide. Australian and New Zealand Journal of Public Health 1996;20:76-82.

\section{Slade 1997}

Slade GD, Gansky SA, Spencer AJ. Two-year incidence of tooth loss among South Australians aged 60+ years. Community Dentistry and Oral Epidemiology 1997;25:429-37.

\section{Slade 2014}

Slade GD, Akinkugbe AA, Sanders AE. Projections of US edentulism prevalence following 5 decades of decline. Journal of Dental Research 2014;93(10):959-65.

\section{Slayton 2018}

Slayton RL, Urquhart O, Araujo MWB, Fontana M, GuzmánArmstrong S, Nascimento MN, et al. Evidence-based clinical practice guideline on nonrestorative treatments for carious lesions: a report from the American Dental Association. Journal of the American Dental Association 2018;149(10):837-49.

\section{Sugihara 2010}

Sugihara N, Maki Y, Okawa Y, Hosaka M, Matsukubo T, Takaesu Y. Factors associated with root surface caries in elderly. Bulletin of Tokyo Dental College 2010;51:23-50.

\section{Tam 2001}

Tam LE, McComb D. Diagnosis of occlusal caries: Part II. Recent diagnostic technologies. Journal of the Canadian Dental Association 2001;67:459-64.

\section{Vos 2016}

GBD 2015 Disease and Injury Incidence and Prevalence Collaborators. Global, regional, and national incidence, prevalence, and years lived with disability for 310 acute and chronic diseases and injuries, 1990-2015: a systematic analysis for the global burden of disease study 2015. Lancet 2016;388(10053):1545-602.

\section{CHARACTERISTICS OF STUDIES}

Characteristics of included studies [ordered by study ID]

\section{Wefel 1995}

Wefel JS, Heillman JR, Jordan TH. Comparisons of in vitro root caries models. Caries Research 1995;29:204-9.

\section{Wenzel 2000}

Wenzel A, Anthonisen PN, Juul MB. Reproducibility in the assessment of caries lesion behaviour: a comparison between conventional film and subtraction radiography. Caries Research 2000;34:214-8.

\section{White 2012}

White D, Tsakos G, Pitts N, Fuller E, Doulgas GVA, Murray JJ, et al. Adult Dental Health Survey 2009: common oral health conditions and their impact on the population. British Dental Journal 2012;213:567-72.

\section{Whiting 2011}

Whiting PF, Rutjes AWS, Westwood ME, Mallett S, Deeks JJ, Reitsma JB, et al. QUADAS-2: a revised tool for the quality assessment of diagnostic accuracy studies. Annals of Internal Medicine 2011;155:529-36.

\section{Wilson 1968}

Wilson JMG, Jungner G, World Health Organization. Principles and Practice of Screening for Disease. Geneva: World Health Organization, 1968.

\section{World Health Organization 1997}

World Health Organization. Oral Health Surveys: Basic Methods. 4th edition. Geneva: World Health Organization, 1997.

\section{World Health Organization 2013}

World Health Organization. Oral Health Surveys: Basic Methods. 5th edition. Vol. 53. Geneva: World Health Organization, 2013.

\section{World Health Organization 2017}

World Health Organization. Sugars and dental caries. WHO Technical Information Note. apps.who.int/iris/bitstream/ handle/10665/259413/WHO-NMH-NHD-17.12-eng.pdf? sequence $=1$ (accessed 15 May 2018).

Neuhaus 2012

\section{Study characteristics}

Patient Sampling

\author{
Method of sampling: selected from a pool of extracted teeth \\ Included conditions: visually sound surfaces or signs of demineralisation \\ Teeth: permanent molars \\ Sealants: no
}

Restorations: all selected teeth had Class II amalgam restorations 
Neuhaus 2012 (Continued)

Surface: approximal root surfaces

Patient characteristics and setting

Age: not reported

Sex: not reported

Ethnicity: not reported

Country: Switzerland

Setting: "a pool of extracted teeth"

Number of participants/teeth/sites: 80 teeth in total, 30 teeth where proximal restoration margins extended onto the root surface, 30 root surfaces

Prevalence: $20 / 30=0.67$

Concern that this study reports secondary caries which may not be applicable to primary caries setting and therefore differs to our review question

Index tests

Category of test: radiograph (bitewing), laser fluorescence pen

Model set up: "The study teeth were mounted in pairs between two sound teeth, whose roots were embedded in composite resin, in order to obtain a tight contact"

Sequence of test(s): index tests carried out prior to reference standard

Examiner training and calibration: no training or calibration, "Two trained examiners assessed the radiographs independently"

Threshold applied: radiographic examination: classified as no radiolucency or radiolucency in dentine; laser fluorescence pen threshold: > 22"

Device specifics: "Bitewing radiographs (BW) were taken of all blocks using X-ray machine (HDX Dental EZ, USA) and double Kodak Insight films $(22 \mathrm{~mm} \times 35 \mathrm{~mm}$, Kodak, Rochester, MN, USA) at $65 \mathrm{kV}, 7 \mathrm{~mA}$ and an exposure time of $0.09 \mathrm{~s}$.The source-to-film distance was $6100 \mathrm{~mm}$. Close to the object on the focus side, a 5-mm-wide plastic mould was placed to simulate soft tissues"; "LF pen measurements were carried out using... 0.4 $\mathrm{mm}$ thickness: TWDG (tapered wedge-shaped) $0.7 \mathrm{~mm}$ width. The device was calibrated before each measurement according to the manufacturer's instructions"

Target condition and reference standard(s)
Category: histology

Sequence of index test and reference standard: reference standard followed all index tests

Training of examiner: not reported, although 2 examiners were used who reached a consensus

Blinding to index test: not reported but assumed to be the case

Multiple tests: no

Site selection: the teeth were ground longitudinally until $1 \mathrm{~mm}$ before the site of measurement

Target condition: caries free or root caries

Flow and timing
Participants with index test but no reference standard: 0

Participants with reference standard but no index test: 0

Time interval between tests: not reported 
Neuhaus 2012 (Continued)

Participants receiving both tests but excluded from results: 0

Comparative

Notes

Data from pre-specified thresholds for tapered wedge-shaped (TWDG) tip have been used in the analyses

\section{Methodological quality}

\begin{tabular}{llll}
\hline Item & Authors' judgement & Risk of bias & Applicability concerns \\
\hline DOMAIN 1: Patient Selection & &
\end{tabular}

Was a consecutive or random sample of No patients enrolled?

\begin{tabular}{ll}
\hline Was a case-control design avoided? & Yes \\
\hline $\begin{array}{l}\text { Did the study avoid inappropriate exclu- } \\
\text { sions? }\end{array}$ & Yes
\end{tabular}

sions?

Could the selection of patients have in-
troduced bias?

Are there concerns that the included
patients and setting do not match the
review question?

\section{DOMAIN 2: Index Test (All tests)}

Were the index test results interpreted

without knowledge of the results of the reference standard?

If a threshold was used, was it pre-speci- Yes fied?

Could the conduct or interpretation of Low risk
the index test have introduced bias?

Are there concerns that the index test,

\section{DOMAIN 3: Reference Standard}

\section{Is the reference standards likely to cor- Yes} rectly classify the target condition?

Were the reference standard results inter- Yes preted without knowledge of the results of the index tests?

\section{Could the reference standard, its con- duct, or its interpretation have intro- duced bias?}


Neuhaus 2012 (Continued)

\section{Are there concerns that the target con- dition as defined by the reference stan- dard does not match the question?}

\section{Low concern}

\section{DOMAIN 4: Flow and Timing}

Was there an appropriate interval be-

Yes

tween index test and reference standard?

Did all patients receive the same refer- $\quad$ Yes

ence standard?

Were all patients included in the analysis? Yes

Could the patient flow have introduced

Low risk

bias?

\section{Rodrigues 2010}

\section{Study characteristics}

Patient Sampling

Method of sampling: selected teeth from a pool of previously extracted molars

Included conditions: Class II composite restorations

Teeth: permanent molars

Sealants: no

Restorations: all selected teeth had Class II composite restorations

Surface: approximal root surfaces

Patient characteristics and setting Age: not reported

Sex: not reported

Ethnicity: not reported

Country: Switzerland

Setting: "teeth had been extracted for periodontal or orthodontic reasons by dental practitioners"

Number of participants/teeth/sites: 43 teeth, 60 surfaces of which 29 were roots

Prevalence: $16 / 29=0.55$

Concern that this study reports secondary caries which may not be applicable to primary caries setting and therefore differs to our review question

Index tests

Category of test: radiograph (bitewing), visual examination, combined visual and radiographic examination, laser fluorescence pen (DIAGNOdent)

Model set up: "The tooth under study was placed between two sound teeth that had their roots embedded in a composite simulating the contact points of adult teeth. Soft tissue was not simulated in the blocks"

Sequence of test(s): in the order listed above, all prior to reference standard, radiograph and visual separated by 3 weeks from combined test 
Examiner training and calibration: no training or calibration, 2 experienced examiners were used

Threshold applied: radiographic examination: classified as no radiolucency or radiolucency in dentine; visual examination: classified as sound surface or root caries (diagnostic criteria not reported); combined visual and radiographic examination: the lowest score from radiographic and from visual examination was registered; threshold for DIAGNOdent was the optimal threshold "the LF pen and VE showed the highest values of specificity (0.54), sensitivity (0.81) and accuracy (0.69) at a cutoff of 18 "

Device specifics: "Bitewings radiographs (BW) were taken of all blocks using an $\mathrm{x}$-ray machine (HDX, DentalEZ, Lancaster, PA, USA) and twin Kodak Insight films $(22 \times 35$ mm, Eastman Kodak Company, Rochester, NY, USA) at $65 \mathrm{kV}, 7 \mathrm{~mA}$ and an exposure time of 0.09 seconds. The source-to-film distance was $6100 \mathrm{~mm}$. A 5-mm wide plastic mold was placed close to the object on the focus side to simulate soft tissue"; "The LF pen (DIAGNOdent 2190, Kavo) measurements were carried out using a wedge-shaped sapphire fiber tip $(1.1 \mathrm{~mm})$ for the approximal surface"; "optimal cutoff values for secondary approximal caries lesions on the crown and root surfaces were determined by the ROC curve at the point for which the sum of the sensitivity and specificity was maximal"

Target condition and reference standard(s)
Category: histology

Sequence of index test and reference standard: reference standard followed all index tests

Training of examiner: not reported, although 2 examiners were used who reached a consensus

Blinding to index test: not reported but assumed to be the case

Multiple tests: no

Site selection: sectioned teeth

Target condition: caries free or root caries

Flow and timing

Participants with reference standard but no index test: 0

Time interval between tests: minimal

Participants receiving both tests but excluded from results: 0

Comparative

Notes

Concern that the scoring system is not reported for visual or radiograph examinations, this is probably an aim to reflect clinical practice but this is an in vitro study. This observation is not reflected in any of the bias or applicability decisions

\section{Methodological quality}

\begin{tabular}{llll}
\hline Item Authors' judgement $\quad$ Risk of bias & Applicability concerns
\end{tabular}

\section{DOMAIN 1: Patient Selection}

Was a consecutive or random sam- No

ple of patients enrolled?

\section{Was a case-control design avoid- Yes}

ed? 
Rodrigues 2010 (Continued)

Did the study avoid inappropriate Yes exclusions?

\section{Could the selection of patients}

have introduced bias?

\section{High risk}

\section{Are there concerns that the in- cluded patients and setting do \\ not match the review question?}

DOMAIN 2: Index Test (All tests)

Were the index test results interpreted without knowledge of the results of the reference standard? If a threshold was used, was it pre- No
specified?

\section{Could the conduct or interpreta-} tion of the index test have introduced bias?

\section{Are there concerns that the in- dex test, its conduct, or inter- pretation differ from the review question?}

\section{DOMAIN 3: Reference Standard}

Is the reference standards likely to correctly classify the target condition?

Were the reference standard results interpreted without knowledge of the results of the index tests?

\section{Could the reference standard, its conduct, or its interpretation have introduced bias?}

\section{Are there concerns that the tar- get condition as defined by the reference standard does not match the question?}

\section{DOMAIN 4: Flow and Timing}

Was there an appropriate interval Yes between index test and reference standard?

Did all patients receive the same 
Rodrigues 2010 (Continued)

Were all patients included in the Yes analysis?

\section{Could the patient flow have in-}

troduced bias?

Low risk

Zhang 2009

\section{Study characteristics}

Patient Sampling

Method of sampling: population of 717 adults was screened, those with 5 exposed

root surfaces were included

Included conditions: adults with at least 5 exposed root surfaces

Teeth: not reported

Sealants: not applicable

Restorations: not reported

Surface: root - buccal and lingual surfaces

Patient characteristics and setting

Age: 60 to 90 years (mean 72.5 )

Sex: not reported

Ethnicity: not reported

Country: Hong Kong

Setting: community setting

Number of participants/teeth/sites: 5324 teeth examined and 4188 had exposed root surfaces, 3781 sound and 198 carious (missing 209)

Prevalence: $198 / 4188=0.05$

Concern that a high number of inactive lesions are present

Sequence of test(s): scaling completed by dental hygienist, then visual-tactile assessment (reference standard), then fluorescence test (index test)

Examiner training and calibration: "trained and calibrated dentist"; "masked of visual-tactile root surface assessments"

Threshold applied: none applied a priori, threshold of 8 produced optimum results (figure 2 in paper)

Device specifics: "The exposed buccal and lingual root surfaces of teeth were measured by DD using a type A tip", the DIAGNOdent value was recorded

\footnotetext{
Target condition and reference standard(s)
}

\section{Category: visual-tactile examination}

Sequence of index test and reference standard: visual-tactile assessment prior to index test so not affected by the results

Training of examiner: not reported 
Zhang 2009 (Continued)

Blinding to index test: yes

Multiple tests: no

Site selection: roots - buccal and lingual surfaces

Target condition: sound - normal texture, no colour change; carious - active: yellowish or light brownish, soft upon probing; carious - inactive: darkly discoloured, smooth, and hard upon light probing (World Health Organization 1997 diagnostic criteria)

Flow and timing

Participants with index test but no reference standard: 0

Participants with reference standard but no index test: 0

Time interval between tests: minimal

Participants receiving both tests but excluded from results: 209 surfaces missing from the results section

\section{Comparative}

\section{Notes}

\section{Methodological quality}

\begin{tabular}{lccc}
\hline Item & Authors' judgement & Risk of bias & Applicability concerns \\
\hline DOMAIN 1: Patient Selection & &
\end{tabular}

Was a consecutive or random sample of pa- Yes

tients enrolled?

\begin{tabular}{ll}
\hline Was a case-control design avoided? & Yes \\
\hline Did the study avoid inappropriate exclusions? & Yes \\
\hline
\end{tabular}

Could the selection of patients have introduced bias?

Low risk

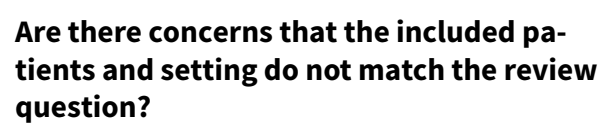

\section{DOMAIN 2: Index Test (All tests)}

\section{Were the index test results interpreted without Yes} knowledge of the results of the reference standard?

\begin{tabular}{ll}
\hline If a threshold was used, was it pre-specified? No & Nigh risk \\
\hline $\begin{array}{l}\text { Could the conduct or interpretation of the } \\
\text { index test have introduced bias? }\end{array}$ & Hian
\end{tabular}

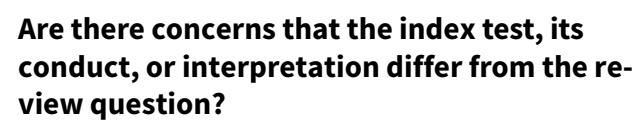


Zhang 2009 (Continued)

\section{DOMAIN 3: Reference Standard}

Is the reference standards likely to correctly No classify the target condition?

Were the reference standard results interpret- Yes ed without knowledge of the results of the index tests?

Could the reference standard, its conduct, or $\quad$ High risk
its interpretation have introduced bias?

its interpretation have introduced bias?

\section{Are there concerns that the target condition as defined by the reference standard does not match the question?}

\section{DOMAIN 4: Flow and Timing}

Was there an appropriate interval between in- Yes dex test and reference standard?

Did all patients receive the same reference Yes standard?

Were all patients included in the analysis? No

Could the patient flow have introduced bias?

High risk

\section{Zhang 2016}

\section{Study characteristics}

Patient Sampling

Method of sampling: "Purposive sampling" subjects with at least 5 exposed root surfaces

Included conditions: exposed roots

Teeth: not reported

Sealants: not applicable

Restorations: no restorations

Surface: root - surface not reported

Age: 60 to 84 years

Sex: 17 male (35\%), 31 female (65\%)

Ethnicity: not reported

Country: Hong Kong

Setting: community screening programme

Number of participants/teeth/sites: 48 participants, 750 teeth

Prevalence: $134 / 750=0.18$ 
Zhang 2016 (Continued)

Index tests

Category of test: fluorescence device - DIAGNOdent (2095)

Sequence of test(s): both index and reference standard completed before and after scaling, results used after scaling completed at the optimum value of sensitivity and specificity

Examiner training and calibration: experienced examiner

Threshold applied: none applied a priori

Device specifics: DIAGNOdent (DD) (2095) - the maximum DD value recorded on the exposed root surface, type-A tip

Target condition and reference standard(s)

Category: visual-tactile examination using Community Periodontal Index (CPI) probe

Sequence of index test and reference standard: both index and reference standard completed before and after scaling, potential for results of examination prior to scaling to effect post-scaling decision. Order of index test and reference standard unclear.

Training of examiner: not reported

Blinding to index test: not reported

Multiple tests: no

Site selection: root - surface not reported

Target condition: sound - normal texture, no colour change; carious - active: yellowish or light brownish, soft upon probing; carious - inactive: darkly discoloured, smooth, and hard upon light probing (Fejerskov 1991 and World Health Organization 2013 diagnostic criteria)

Flow and timing

Participants with index test but no reference standard: 0

Participants with reference standard but no index test: 0

Time interval between tests: minimal

Participants receiving both tests but excluded from results: 0

\section{Comparative}

\section{Notes}

Results derived from text. 134 reported as carious after scaling therefore 616 were sound; and the quote "A DD cut-off value between 5 and 10 produced the highest combined sensitivity and specificity for root caries, with both the sensitivity and specificity at around $80 \% "$

\section{Methodological quality}

\begin{tabular}{llll}
\hline Item & Authors' judgement & Risk of bias & Applicability concerns \\
\hline
\end{tabular}

DOMAIN 1: Patient Selection

Was a consecutive or random sample of pa- No tients enrolled?

\begin{tabular}{ll}
\hline Was a case-control design avoided? & Yes \\
\hline Did the study avoid inappropriate exclusions? & Yes \\
\hline
\end{tabular}


Zhang 2016 (Continued)

Could the selection of patients have intro-

High risk duced bias?

Are there concerns that the included pa-

Low concern tients and setting do not match the review question?

\section{DOMAIN 2: Index Test (All tests)}

Were the index test results interpreted without Unclear knowledge of the results of the reference standard?

If a threshold was used, was it pre-specified?

No

Could the conduct or interpretation of the High risk
index test have introduced bias?

Are there concerns that the index test, its conduct, or interpretation differ from the review question?

\section{DOMAIN 3: Reference Standard}

Is the reference standards likely to correctly No

classify the target condition?

Were the reference standard results interpret- Unclear ed without knowledge of the results of the index tests?

Could the reference standard, its conduct, or its interpretation have introduced bias?

\section{Are there concerns that the target condition as defined by the reference standard does not match the question?}

\section{DOMAIN 4: Flow and Timing}

Was there an appropriate interval between in- Yes dex test and reference standard?

Did all patients receive the same reference Yes standard?

Were all patients included in the analysis? Yes 


\begin{tabular}{|c|c|}
\hline Study & Reason for exclusion \\
\hline Amaechi 2004 & $\begin{array}{l}\text { Artificial caries and not a DTA study (assessing correlation between artificial root caries lesion } \\
\text { depth and reflectivity of OCT images) }\end{array}$ \\
\hline Baysan 2004 & No reference standard \\
\hline Darling 2012 & Not a DTA study, no reference standard, and no sound teeth \\
\hline Ekstrand 2007 & Artificial root caries and not a DTA study \\
\hline Fried 2002 & Not a DTA study, no sound teeth \\
\hline Galal 1985 & $\begin{array}{l}\text { No reference standard. Root caries and coronal caries not reported separately and unclear if root } \\
\text { caries is included as a target condition of this study }\end{array}$ \\
\hline Karlsson 2009 & $\begin{array}{l}\text { Not a DTA study (assessing correlation between laser fluorescence readings and histological depth, } \\
\text { colour, and texture of root caries lesions) }\end{array}$ \\
\hline Lee 2017 & $\begin{array}{l}\text { Not a DTA study (assessing mean values of PS-OCT reflectivity and thermal readings for active and } \\
\text { arrested root caries lesions) }\end{array}$ \\
\hline Lynch 1994 & No sound teeth \\
\hline Mello 2011 & No sound teeth \\
\hline Nordenram 1988 & Data not reported in a format that allows a $2 \times 2$ table to be created \\
\hline Ratanaporncharoen 2018 & Not a DTA study, no sound teeth \\
\hline Sikri 1991 & Data not reported in a format that allows a $2 \times 2$ table to be created \\
\hline Wicht 2002 & $\begin{array}{l}\text { Not a DTA study (assessing correlation between root caries lesion colour, texture, and cavitation } \\
\text { depth and laser fluorescence readings and ECM scores) }\end{array}$ \\
\hline Zhou 2018 & $\begin{array}{l}\text { Not a DTA study, methods allow the authors to analyse dentine lesion shrinkage, does not report } \\
\text { data in a format that allows a } 2 \times 2 \text { table to be used. Also used sound roots and created artifical } \\
\text { caries }\end{array}$ \\
\hline
\end{tabular}

DTA = diagnostic test accuracy; ECM = electronic caries monitor; OCT = optical coherence tomography; PS-OCT = polarization sensitive optical coherence tomography.

\section{ADDITIONAL TABLES}

Table 1. Classification of levels of caries

\begin{tabular}{ll}
\hline DMFT classification & Definition (Pitts 2001) \\
\hline 0 & Sound (non-diseased) \\
\hline$D_{1}$ & Non-cavitated yet clinically detectable enamel lesions with intact surfaces \\
\hline$D_{2}$ & Cavitated lesion penetrating the enamel or shadowing \\
\hline$D_{3}$ & Cavity progressing past the enamel-dentine junction into dentine \\
\hline
\end{tabular}


Table 1. Classification of levels of caries (Continued)

$\mathrm{D}_{4}$

Cavity progressing into pulp

DMFT = decayed, missing, and filled teeth scale.

Table 2. Index tests for root caries

$\begin{array}{ll}\text { Test Characteristics } & \begin{array}{l}\text { Intended use in clin- } \\ \text { ical pathway }\end{array}\end{array}$

Visual or visu- Identifying root caries according to visual apal-tactile exam- pearance or texture, aided by a dental mirror ination
The fundamental step in the detection of root caries, but limited in the diagnosis of early noncavitated lesions. All patients presenting to a dental clinician will receive a visual examination
Advantages: completed and interpreted quickly with minimal invasion and little cost except clinician training and time

Disadvantages: early or approximal lesions are difficult to observe visually, difficult to monitor lesions longitudinally with visual-tactile methods

\begin{abstract}
Radiography
Bitewing radiology is the most commonly used method. Others include: subtraction radiographs which provides a semi-automated method for monitoring progression of lesions (Ellwood 1997; Wenzel 2000) and cone beam computed technology (CBCT) which provides a 3-dimensional image which appears to offer great potential for diagnosis with increased levels of radiation (Horner 2009)
\end{abstract}

Used as an adjunct to aid detection of approximal lesions difficult to assess visually
Advantages: radiographs may aid in the detection of approximal cavitated root caries lesions difficult to detect with visual-tactile examination alone (Rodrigues 2011)

Disadvantages: limitations exist when detecting early, non-cavitated lesions. Cervical burnout presenting as proximal radiolucency can complicate accurate detection of root caries radiographically. There is a small but real risk over patient exposure to ionizing radiation, which has to be balanced with the patient's age, caries risk, and time since previous radiograph (Pitts 2017)

\footnotetext{
Fluorescence Caries-induced changes in teeth alter the characteristics of its structure, causing diseased teeth to respond differently to sound teeth when exposed to light-inducing fluorescence. There is potential for mineral loss to be quantified and used to aid the diagnostic decision and treatment pathway (Angmar-Månsson 2001; Matos 2011; Wicht 2002). Fluorescence is typically divided into laser fluorescence and light fluorescence (i.e. DIAGNOdent type devices and quantitative light-induced fluorescence (QLF) type devices)
}

Potential to aid the clinician in identifying early caries which may not be detected with a visual examination alone
Advantages: the potential to identify changes in tooth characteristics that are otherwise unobservable in a visual-tactile examination

Disadvantages: uncertainty of the reliability of devices and the ability to detect disease and health
FOTI uses a light emitted from a handheld device which when placed directly onto the tooth illuminates the tooth (Pretty 2006). Any demineralisation should appear as shadows in the tooth due to the disruption of the tooth's structure due to caries
An adjunct to the visual examination, particularly useful for detecting early approximal caries (Davies 2001). A further advancement with fibre-optic techniques combines
Advantages: the potential to identify changes in tooth characteristics that are otherwise unobservable in a visual-tactile examination

Disadvantages: uncertainty of the reliability of devices and the ability to detect disease and health 
Table 2. Index tests for root caries (Continued)

\section{this with a camera to capture an image which may or may not be linked to soft- ware for analysis, digital imaging FOTI (DIFOTI)}

Electrical con- The demineralisation of the tooth is reportductance ed to have an effect on the tooth's electrical conductance. This is measured by placing a probe on the tooth which measures any potentially higher conductivity which occurs due to carious lesions being filled with saliva (Tam 2001)
An adjunct to the visual examination
Advantages: the potential to identify changes in tooth characteristics that are otherwise unobservable in a visual-tactile examination

Disadvantages: uncertainty of the reliability of devices and the ability to detect disease and health. Particularly due to the necessity to place the probe in an identical location for a reproducible result

Table 3. QUADAS-2 tool

Item Response (delete as required)

\section{Participant selection - Risk of bias}

1) Was a consecutive or random sample of participants or teeth used?
Yes - where teeth or participants were selected consecutively or allocated to the study via a randomisation process

No - if study described another method of sampling

Unclear - if participant sampling is not described
2) Was a case-control design avoided?

Yes - if case-control clearly not used

No - if study described as case-control or describes sampling specific numbers of participants with particular diagnoses

Unclear - if not clearly described

3) Did the study avoid inappropriate exclusions (e.g. inclusion of caries into dentine)?
Yes - if the study clearly reports that included participants or teeth were apparently healthy or caries into dentine were excluded

No - if lesions were included that showed caries into dentine or exclusions that might affect test accuracy (e.g. teeth with no caries)

Unclear - if not clearly reported

\section{Could the selection of participants have introduced bias?}

If answers to all of questions 1) and 2) and 3) Risk is low
was 'yes'

\footnotetext{
If answers to any of questions 1) and 2) and 3) Risk is high was 'no'
}
If answers to any of questions 1) and 2) and 3) Risk is unclear was 'unclear'


Table 3. QUADAS-2 tool (Continued)

\section{Participant selection - Concerns regarding applicability}

1) Does the study report results for participants or teeth selected by apparent health or suspected early caries (i.e. studies do not recruit patients who are known to have advanced caries into dentine)?
Yes - if a group of participants or teeth has been included which is apparently healthy or indicative of early caries

No - if a group of participants or teeth has been included which is suspected of advanced caries

Unclear - if insufficient details are provided to determine the spectrum of participants or teeth

Yes - if the analysis was reported on a surface or tooth basis

No - if the analysis was reported on a per-patient basis

Unclear - if it is not possible to assess whether data are presented on a per-patient or per-tooth basis

Yes - if the participants were recruited prior to tooth extraction

3) Did the study avoid an in vitro setting which required the usage of extracted teeth?

Unclear - if it was not possible to assess the source and method of recruiting of included participants/teeth

\section{Is there concern that the included participants or teeth do not match the review question?}

If answers to all of questions 1) and 2) and 3) Risk is low
was 'yes'

\section{If answers to any of questions 1) and 2) and 3) Risk is high}

was 'no'

If answers to any of questions 1) and 2) and 3) Risk is unclear

was 'unclear'

\section{Index test - Risk of bias (to be completed per test evaluated)}

1) Was the index test result interpreted without knowledge of the results of the reference standard?

Yes - if the index test described is always conducted and interpreted prior to the reference standard result, or for retrospective studies interpreted without prior knowledge of the reference standard

No - if index test described as interpreted in knowledge of reference standard result

Unclear - if index test blinding is not described

2) Was the diagnostic threshold at which the test was considered positive pre-specified?

Yes - if threshold was pre-specified (i.e. prior to analysing the study results)

No - if threshold was not pre-specified

Unclear - if not possible to tell whether or not diagnostic threshold was pre-specified

\section{For visual and radiograph tests only:}

3) For studies reporting the accuracy of multiple diagnostic thresholds for the same index test or multiple index tests, was each threshold or index test interpreted without knowledge of the results of the others?
Yes - if thresholds or index tests were selected prospectively and each was interpreted by a different clinician or interpreter, or if study implements a retrospective (or no) cut-off (i.e. look for deepest/most severe lesion first)

No - if study states reported by same reader

Unclear - if no mention of number of readers for each threshold or if pre-specification of threshold not reported 
Table 3. QUADAS-2 tool (Continued)

N/A - multiple diagnostic thresholds not reported for the same index test

\section{Could the conduct or interpretation of the index test have introduced bias?}

\section{For visual and radiographic studies item 3 ) to be added}

If answers to all of questions 1) and 2) was Risk is low

'yes'

If answers to any of questions 1) and 2) was Risk is high

'no'

If answers to any of questions 1) and 2) was

'unclear'

Risk is unclear

\section{Index test - Concerns regarding applicability}

1) Were thresholds or criteria for diagnosis reported in sufficient detail to allow replication?
Yes - if the criteria for detection or diagnosis of the target disorder were reported in sufficient detail to allow replication

No - if the criteria for detection or diagnosis of the target disorder were not reported in sufficient detail to allow replication

Unclear - if some but not sufficient information on criteria for diagnosis to allow replication were provided

Yes - if the test clearly reported that the test was interpreted by an experienced examiner

No - if the test was not interpreted by an experienced examiner

Unclear - if the experience of the examiner(s) was not reported in sufficient detail to judge or if examiners described as 'Expert' with no further detail given

\section{Is there concern that the included participants do not match the review question?}

\begin{tabular}{ll}
\hline If the answer to question 1) and 2) was 'yes' & Concern is low \\
\hline If the answer to question 1) and 2) was 'no' & Concern is high \\
\hline $\begin{array}{l}\text { If the answer to question 1) and 2) was 'un- } \\
\text { clear' }\end{array}$ & \\
\hline
\end{tabular}

\section{Reference standard - Risk of bias}

1) Is the reference standard likely to correctly Yes - if all teeth or surfaces underwent a histological or excavation reference stanclassify the target condition? dard

No - if a final diagnosis for any participant or tooth was reached without the histological or excavation reference standards

Unclear - if the method of final diagnosis was not reported

2) Were the reference standard results interpreted without knowledge of the results of the index test?
Yes - if the reference standard examiner was described as blinded to the index test result

No - if the reference standard examiner was described as having knowledge of the index test result

Unclear - if blinded reference standard interpretation was not clearly reported 
Table 3. QUADAS-2 tool (Continued)

Could the reference standard, its conduct, or its interpretation have introduced bias?

\begin{tabular}{ll}
\hline If answers to questions 1) and 2) was 'yes' & Risk is low \\
\hline If the answer to question 1) and 2) was 'no' & Concern is high \\
\hline
\end{tabular}

If the answer to question 1) and 2) was 'un- Concern is unclear clear'

\section{Reference standard - Concerns regarding applicability}

1) Does the study use the same definition of disease positive as the prescribed in the review question?
Yes - same definition of disease positive used, or teeth can be disaggregated and regrouped according to review definition

No - some teeth cannot be disaggregated

Unclear - definition of disease positive not clearly reported

\section{Flow and timing - Risk of bias}

1) Was there an appropriate interval between index test and reference standard (in vivo studies less than 3 months, in vitro no limit but must be stored appropriately)?
Yes - if study reports index and reference standard had a suitable interval or storage method

No - if study reports greater than 3-month interval between index and reference standard or inappropriate storage of extracted teeth prior to reference standard

Unclear - if study does not report interval or storage methods between index and histological reference standard

Yes - if all participants underwent the same reference standard

No - if more than 1 reference standard was used

Unclear - if not clearly reported
2) Did all participants receive the same reference standard?
Yes - if all participants were included in the analysis

No - if some participants were excluded from the analysis

Unclear - if not clearly reported

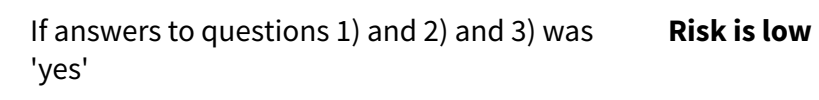

Risk is unclear

N/A = not applicable; QUADAS-2 = Quality Assessment of Diagnostic Accuracy Studies 2.

\section{H I S T ORY}

Review first published: Issue 12, 2020

\section{CONTRIBUTIONS OF AUTHORS}

All review authors collaborated in the conception of the review purpose and design. Drafting the protocol: Richard Macey (RM) and Tanya Walsh (TW). 
Developing the search strategy: TW and RM.

Screening of papers against eligibility criteria: Patrick Fee (PF) and RM.

Appraising the quality of papers: PF and RM.

Extracting data for the review: PF and RM.

Entering data into Review Manager 5 (Review Manager 2020): PF and RM.

Analysis of data: PF, RM, and TW.

Drafting the review: all authors.

\section{DECLARATIONS OF INTEREST}

Patrick A Fee: none known.

Richard Macey: none known.

Tanya Walsh: none known. I am Statistical Editor with Cochrane Oral Health.

Janet E Clarkson: none known. I am Joint Co-ordinating Editor of Cochrane Oral Health.

David Ricketts: none known.

\section{SOURCES OF SUPPORT}

\section{Internal sources}

- Division of Dentistry, School of Medical Sciences, Faculty of Biology, Medicine and Health, The University of Manchester, UK

- Manchester Academic Health Sciences Centre (MAHSC) and the NIHR Manchester Biomedical Research Centre, UK

\section{External sources}

- National Institute for Health Research (NIHR), UK

NIHR Cochrane Programme Grant: 16/114/23 Detection and diagnosis of common oral diseases: diagnostic test accuracy of tests of oral cancer and caries

- NIHR, UK

This project was supported by the NIHR, via Cochrane Infrastructure funding to Cochrane Oral Health. The views and opinions expressed herein are those of the review authors and do not necessarily reflect those of the Evidence Synthesis Programme, the NIHR, the NHS, or the Department of Health and Social Care

- Cochrane Oral Health Global Alliance, Other

The production of Cochrane Oral Health reviews has been supported financially by our Global Alliance since 2011 (oralhealth.cochrane.org/partnerships-alliances). Contributors in the last 2 years have been the American Association of Public Health Dentistry, USA; AS-Akademie, Germany; the British Association for the Study of Community Dentistry, UK; the British Society of Paediatric Dentistry, UK; the Canadian Dental Hygienists Association, Canada; the Centre for Dental Education and Research at All India Institute of Medical Sciences, India; the National Center for Dental Hygiene Research \& Practice, USA; New York University College of Dentistry, USA; and Swiss Society of Endodontology, Switzerland 